\title{
WHAT SHAPES THE FAR-INFRARED SPECTRAL ENERGY DISTRIBUTIONS OF GALAXIES?
}

\author{
Mohammadtaher Safarzadeh ${ }^{1}$, Christopher C. Hayward ${ }^{2,3,6}$, Henry C. Ferguson ${ }^{4}$, and Rachel S. Somerville ${ }^{5}$ \\ ${ }^{1}$ Johns Hopkins University, Department of Physics and Astronomy, 366 Bloomberg Center, 3400 N. Charles Street, Baltimore, MD 21218, USA; mts@ pha.jhu.edu \\ 2 TAPIR 350-17, California Institute of Technology, 1200 E. California Boulevard, Pasadena, CA 91125, USA \\ ${ }^{3}$ Harvard-Smithsonian Center for Astrophysics, 60 Garden Street, Cambridge, MA 02138, USA \\ ${ }^{4}$ Space Telescope Science Institute, 3700 San Martin Boulevard, Baltimore, MD 21218, USA \\ ${ }^{5}$ Department of Physics and Astronomy, Rutgers, The State University of New Jersey, 136 Frelinghuysen Road, Piscataway, NJ 08854, USA \\ Received 2015 August 31; accepted 2015 December 22; published 2016 February 8
}

\begin{abstract}
To explore the connection between the global physical properties of galaxies and their far-infrared (FIR) spectral energy distributions (SEDs), we study the variation in the FIR SEDs of a set of 51 hydrodynamically simulated galaxies, both mergers and isolated systems representative of low- and high-redshift galaxies, that are generated by performing dust radiative transfer in post-processing. We study the FIR SEDs using principal component (PC) analysis, and find that $97 \%$ of the variance in the sample can be explained by two PCs. The first PC characterizes the wavelength of the peak of the FIR SED, and the second encodes the breadth of the SED. We find that the coefficients of both PCs can be predicted well using a double power law in terms of the IR luminosity and dust mass, which suggests that these two physical properties are the primary determinants of galaxies' FIR SED shapes. Incorporating galaxy sizes does not significantly improve our ability to predict the FIR SEDs. Our results suggest that the observed redshift evolution in the effective dust temperature at a fixed IR luminosity is not driven by geometry: the SEDs of $z \sim 2-3$ ultraluminous IR galaxies (ULIRGs) are cooler than those of local ULIRGs, not because the high-redshift galaxies are more extended, but rather because they have higher dust masses at fixed IR luminosity. Finally, based on our simulations, we introduce a two-parameter set of SED templates that depend on both IR luminosity and dust mass.
\end{abstract}

Key words: dust, extinction

\section{INTRODUCTION}

Understanding what drives the variation in the far-infrared (FIR) spectral energy distributions (SEDs) of galaxies is a key goal if we wish to maximize the physical insight that can be gleaned from the wealth of data in the era of Herschel (Pilbratt et al. 2010) and the Atacama Large Millimeter Array (ALMA). Unfortunately, interpreting FIR SEDs is difficult because spatially resolved data are not available for the vast majority of galaxies (although ALMA will help greatly in this regard). Moreover, there are various degeneracies (e.g., between the dust temperature distribution and the power-law index of the dust emissivity curve; see Kelly et al. 2012 and references therein) that are difficult to break.

FIR SEDs are fit using a wide variety of methods (see Casey et al. 2014 for a recent review). Often, one or a sum of a few modified blackbody SEDs are used to fit FIR SEDs, but the physical information that can be gained from such models is limited (see, e.g., Hayward et al. 2012 and Smith et al. 2013 for detailed discussions). Another common approach is to use templates: the IR luminosity can be estimated by fitting the available photometry with an IR SED template. Various empirically based, single-parameter templates are widely used (e.g., Chary \& Elbaz 2001; Pope et al. 2008; Rieke et al. 2009; Magdis et al. 2012), although the limitations of this approach due to the luminosity dependence of the shape of FIR SEDs have long been recognized in the literature (e.g., Chapman et al. 2004).

Although empirically based FIR SED templates have many practical uses, physical models are necessary if one wishes to learn about the radiation field, dust properties of a galaxy, and

\footnotetext{
${ }^{6}$ Moore Prize Postdoctoral Scholar in Theoretical Astrophysics.
}

the geometry of dust with respect to the radiation sources. Because of the computational expense of radiative transfer and the difficulty of constraining many free parameters with a limited number of FIR photometric data points, approaches to modeling FIR SEDs without doing radiative transfer calculations have been advocated (e.g., Desert et al. 1990; Devriendt et al. 1999; Dale et al. 2001; Dale \& Helou 2002; Draine \& Li 2007; da Cunha et al. 2008; Somerville et al. 2012). Some authors (e.g., Dale et al. 2001; Dale \& Helou 2002) model FIR SEDs by assuming that the mass distribution of dust that is exposed to radiation fields of different intensities can be described by a truncated power law. Draine \& Li (2007) expanded this model by adding a delta function to the intensity distribution (at the minimum intensity) to represent diffuse interstellar medium (ISM) dust (see also Draine et al. 2007). Such models can provide good fits to observed SEDs. Similarly, other authors (e.g., Kovács et al. 2010; Magnelli et al. 2012) parameterize SEDs by assuming a power-law distribution of dust temperatures.

Radiative transfer calculations, either with simplified assumptions about the geometry of dust with respect to stars (Witt \& Gordon 1996, 2000; Silva et al. 1998; Efstathiou et al. 2000; Gordon et al. 2001; Takagi et al. 2003; De Geyter et al. 2014), active galactic nuclei (AGNs; e.g., Fritz et al. 2006; Siebenmorgen \& Krügel 2007; Stalevski et al. 2012), or more complicated geometries (Dopita et al. 2005; Popescu et al. 2011; De Looze et al. 2012, 2014; De Geyter et al. 2015), have been qualitatively successful in producing SEDs similar to those observed. It is also possible and (has become increasingly common) to "forward-model" FIR SEDs by performing three-dimensional (3D) dust radiative transfer on the outputs of hydrodynamical simulations of galaxies in post-processing (e.g., Jonsson 2006; Jonsson 
et al. 2006, 2010; Chakrabarti et al. 2007, 2008; Chakrabarti \& Whitney 2009; Narayanan et al. 2010a, 2010b; Hayward et al. 2011, 2012, 2014a; Snyder et al. 2013; DomínguezTenreiro et al. 2014; Lanz et al. 2014; Martínez-Galarza et al. 2014; Granato et al. 2015).

Because explicit radiative transfer calculations can best capture complicated source and dust geometries; directly treat dust absorption (including not only absorption of primary radiation from stars and AGNs but also dust self-absorption), scattering, and re-emission; and fully characterize the $3 \mathrm{D}$ distribution of dust temperature, which depends on both the local radiation field and the grain properties, they provide the best tool for studying how FIR SEDs depend on galaxy properties. By performing 3D Monte Carlo radiative transfer calculations for idealized geometries (i.e., not outputs of hydrodynamical simulations), Misselt et al. (2001) studied the dependence of the shape of the FIR SED on the global parameters of the emitting regions. They found that higher dust mass leads to colder SEDs when the other parameters in their model are fixed. This is a simple consequence of thermal equilibrium. Moreover, a "shell" (a.k.a., foreground screen) geometry results in broader SEDs compared to a geometry in which stars and dust are mixed (which they term the "dusty" geometry) because of differences in the temperature structures of the two models.

Performing radiative transfer on hydrodynamical simulations of galaxy formation has the benefit of including more realistic source and dust geometries than simpler approaches, such as that of Misselt et al. (2001). This approach has been demonstrated (Jonsson et al. 2010) to yield SEDs that agree well with observed local samples of normal star-forming galaxies from the Spitzer Infrared Nearby Galaxies Survey (SINGS; Kennicutt et al. 2003) and local luminous infrared (IR) galaxies (LIRGs) from the Great Observatories All-sky LIRG Survey (GOALS; Armus et al. 2009). Moreover, Lanz et al. (2014) have shown that the SEDs of local interacting galaxies from the Spitzer Interacting Galaxies Survey (SIGS; Lanz et al. 2013; Brassington et al. 2015) can be fit reasonably well with SEDs predicted in this manner; this is also the case for $24 \mu \mathrm{m}$ selected galaxies at $z \sim 0.3-2.8$ (E. J. Roebuck et al. 2016, in preparation). Unfortunately, performing 3D radiative transfer on hydrodynamical simulations is orders of magnitude more computationally expensive than less detailed approaches that assume smooth axisymmetric geometries (e.g., Silva et al. 1998): the former typically requires of order $10-10^{3}$ CPU-hours per galaxy, whereas the latter requires at most a few minutes. Thus, it is instructive to determine whether the high computational expense of performing 3D dust radiative transfer on hydrodynamical simulations of galaxies can be avoided, as it may be possible to characterize the variation among the simulated SEDs using only a few global parameters.

In this work, we investigate whether we can predict the FIR SEDs of simulated galaxies, which were calculated using 3D dust radiative transfer in previous works, using a simple, computationally inexpensive method: principal component analysis (PCA). In our case, PCA yields FIR SED eigenvectors (principal components; hereafter PCs) such that linearly combining them with the mean SED of the sample can capture the variance in the simulated SEDs. The coefficients of each PC are different for each galaxy, and studying how the coefficients depend on global parameters, such as the star-formation rate
(SFR) or IR luminosity, can give physical intuition regarding what drives dispersion in the FIR SEDs.

The simulated galaxy SEDs used in this work have been analyzed extensively in previous works. These or similar simulations have been demonstrated to exhibit good agreement with the SEDs/colors of various classes of real galaxies, including local "normal" galaxies (Jonsson et al. 2010) and (U) LIRGs (Younger et al. 2009; Jonsson et al. 2010; Lanz et al. 2014), high-redshift dusty star-forming galaxies (Narayanan et al. 2010a, 2010b; Hayward et al. 2011, 2012; E. J. Roebuck et al. 2016, in preparation), obscured AGNs (Snyder et al. 2013; E. J. Roebuck et al. 2016, in preparation), post-starburst galaxies (Snyder et al. 2011), and compact quiescent galaxies (Wuyts et al. 2010). Thus, although the simulations used herein naturally have some associated limitations (see Section 8.6), they have the advantage of being well-tested and in agreement with many observational constraints. Furthermore, to the best of our knowledge, they still represent the state-of-the-art in terms of ultraviolet (UV)millimeter (mm) SEDs generated from 3D hydrodynamical simulations of galaxies.

In addition to determining what physical insights about galaxies can be gained from FIR SEDs, we hope to identify possibilities for improving how semi-analytic models (SAMs) of galaxy formation predict FIR SEDs. Some SAMs (e.g., Silva et al. 1998; Granato et al. 2000) employ radiative transfer calculations that assume axisymmetric geometries or analytic models that are designed to capture the results of such calculations (e.g., González et al. 2011). Such calculations have been widely employed, but it is unclear how well their results agree with those of the $3 \mathrm{D}$ radiative transfer calculations performed on hydrodynamical simulations of galaxies with more complex geometries. Other SAMs (e.g., Somerville et al. 2012) use empirically derived templates that are a function of a single parameter, $L_{\mathrm{IR}}$, to predict FIR SEDs. However, such an approach is insufficient; for example, it cannot capture the redshift evolution of the effective dust temperature-IR luminosity relation (Casey et al. 2014 and references therein) by construction. Thus, we aim to determine one or more additional physical parameters, besides $L_{\mathrm{IR}}$, that can be used to predict the FIR SEDs of galaxies. Having determined what additional parameter(s) is necessary to predict the variation in IR SEDs, we can then generate a set of multiparameter SED templates. By incorporating these templates in a SAM, we may be able to resolve the tension between the observed FIR and submillimeter number counts and those predicted by the SAM (Niemi et al. 2012; Somerville et al. 2012); this will be explored in a future work. These templates could be also used in semi-empirical models (e.g., Béthermin et al. 2012, 2013; Bernhard et al. 2014). Finally, these twoparameter templates will be useful for fitting observed SEDs to infer the total IR luminosity and predict fluxes at wavelengths for which data are not available.

The remainder of this paper is organized as follows: in Section 2 we describe the properties of the simulated galaxy SED data set that we use and summarize the details of the hydrodynamical simulation and dust radiative transfer calculation methods. Section 3 summarizes the PCA technique and how we use the PCA results to predict the simulated galaxies' SEDs. In Section 4, we present the results of the PCA of the simulated galaxy SEDs. Section 5 demonstrates that the dust mass is a key parameter, in addition to the IR luminosity, that 
determines the SED shape; whereas in Section 6, we show that incorporating the galaxy size does not improve our ability to predict the FIR SEDs. In Section 7, we present a two-parameter family of SED templates that depend on the IR luminosity and dust mass. In Section 8, we discuss some observational support for the importance of dust mass in determining the SED shape; speculate regarding the unimportance of galaxy size, origin of catastrophic failures to predict the SEDs, and possible implications of using the proposed two-parameter templates in SAMs; and highlight some limitations of our work. Section 9 lists our primary conclusions.

\section{SIMULATED GALAXY SED DATA SET}

In this work, we analyze two sets of FIR SEDs of simulated isolated and merging disk galaxies that were presented in previous works. We first summarize the properties of the two data sets and then briefly discuss the simulation methodology. The first SED data set was originally presented in Lanz et al. (2014) and Hayward et al. (2014a). The four progenitor galaxies span a stellar (baryonic) mass range of $6 \times 10^{8}-4 \times 10^{10} M_{\odot}\left(10^{9}-5 \times 10^{10} M_{\odot}\right)$, and their properties were selected to be representative of typical star-forming galaxies in the $z \sim 0$ universe (see Cox et al. 2008 for details). Each of the four progenitors was simulated in isolation, and the binary mergers of all possible combinations of progenitors were simulated for a single representative orbit (i.e., the results for other "non-special" orbits are similar; the results for perfectly co-planar and other rare special configurations can sometimes differ significantly; e.g., Cox et al. 2008), thereby yielding 10 merger simulations. The total data set contains $\sim 12,000$ SEDs, and we refer to it as the $z \sim 0$ data set.

The second set of SEDs of simulated isolated disk and merging galaxies was presented in Hayward et al. (2011, 2012, $2013 \mathrm{~b}$ ). For this data set, the structural properties of the progenitor (bulgeless) disk galaxies were scaled to $z \sim 3$ following the method of Robertson et al. (2006), and the initial gas fractions of the disks, 0.6-0.8, are significantly greater than those of the $z \sim 0$ simulations. $^{7}$ Because the original purpose of these simulations was to model high-redshift submillimeter galaxies (SMGs), the progenitor disks span a relatively narrow baryonic mass range of $\sim 1-4 \times 10^{11} M_{\odot}$, but a variety of merger orbits and mass ratios are included (see Hayward et al. 2012 for details). This data set, which we refer to this as the $z \sim 3$ data set, contains 37 hydrodynamical simulations, from which $\sim 6500$ SEDs were calculated.

The SEDs contained in the two data sets were generated by performing dust radiative transfer in post-processing on the outputs of 3D hydrodynamical simulations. The full methodology is described in the aforementioned works and references therein, so we only summarize it here. First, idealized isolated (i.e., non-cosmological) galaxy models were created following the method described in Springel et al. (2005). Each initial disk galaxy is composed of a dark matter halo, stellar and gaseous disks, and a supermassive black hole; for the $z \sim 0$ simulations

\footnotetext{
7 Such large gas fractions were used to ensure that the gas fraction at the time of coalescence was consistent with observational constraints for $z \sim 2$ galaxies. For all snapshots considered in this work, the gas fractions are $<0.5$. See Hayward et al. (2013b) for more details.

8 The traditional density-entropy formulation of SPH was used. However, we note that the results of idealized non-cosmological galaxy simulations such as these are relatively insensitive to the numerical method (Hayward et al. 2014b) and thus do not consider the use of traditional SPH to be a significant limitation.
}

only, a stellar bulge is also included. Then, the isolated galaxies and binary mergers of these galaxies were simulated using a heavily modified version of the GADGET- $2 N$-body/smoothedparticle hydrodynamics (SPH) code (Springel 2005). ${ }^{8}$ The simulations include the effects of gravity, hydrodynamical interactions, and radiative heating and cooling. Star formation and stellar feedback are incorporated via the two-phase subresolution ISM model of Springel \& Hernquist (2003), and BH accretion and AGN feedback are treated following Springel et al. (2005). Each gas particle is enriched with metals according to its associated SFR, assuming a yield of 0.02 .

Every $10 \mathrm{Myr}$, "snapshots" of the physical state of the simulations were saved. Then, to calculate UV-mm SEDs, 3D dust radiative transfer was performed in post-processing on a subset of the snapshots using the code SUNRISE (Jonsson 2006; Jonsson et al. 2010). For a given snapshot, the SUNRISE calculation proceeds as follows: the stellar and $\mathrm{BH}$ particles in the GADGET-2 simulation, which are the sources of radiation, are assigned source SEDs according to their properties (age and metallicity for the star particles and luminosity for the $\mathrm{BH}$ particles). The metal distribution from the simulation is projected onto an octree grid for the purpose of calculating the dust optical depths (assuming a dust-to-metal density ratio of 0.4; e.g., Dwek 1998; James et al. 2002; Sparre et al. 2014). The Milky Way $R_{V}=3.1$ dust model of Draine \& Li (2007) was used.

The most significant uncertainty (of which we are aware) in these calculations is the sub-resolution structure of the ISM. Specifically, the simulations do not resolve the ISM structure on scales of less than a few hundred parsecs, but observations clearly indicate that the ISM has substantial structure on smaller scales. We can crudely investigate this uncertainty through the use of two alternate treatments for the subresolution ISM. ${ }^{9}$ In the first treatment (referred to as "multiphase on" or "default ISM" in previous works), it is assumed that the cold clouds implicit in the Springel \& Hernquist (2003) model have negligible volume filling factors. Thus, for the purpose of the radiative transfer calculations, this dust is ignored. To account for the obscuration of young stars from their birth clouds, a sub-resolution model for the $\mathrm{H}_{\text {II }}$ and photodissociation regions (Groves et al. 2008) is used. In the second treatment ("multiphase off" or "alternate ISM"), the entire dust mass (i.e., dust in both the implicit diffuse phase and cold clouds) is considered in the radiative transfer calculations. In this work, we use the "multiphase off" model to ensure that all dust emission is calculated self-consistently (i.e., there is no component from the sub-resolution $\mathrm{H}_{\text {II }}$ and photodissociation region model). However, we checked that our conclusions are qualitatively unchanged if the "multiphase on" model is used.

After the source and dust properties are specified, radiation transfer is performed using the Monte Carlo method to calculate the effects of dust absorption, scattering, and re-emission. Importantly, dust self-absorption is treated using an iterative procedure in which the transfer of IR radiation and dust temperature calculation are repeated until the luminosity absorbed in each cell is sufficiently converged. For each snapshot, this process yields spatially resolved UV-mm SEDs of the simulated galaxy/merger viewed from seven viewing angles.

\footnotetext{
9 This issue has been discussed extensively in previous works (e.g., Hayward et al. 2011; Snyder et al. 2013; Lanz et al. 2014; Hayward \& Smith 2015), and we refer the interested reader to those papers for additional details.
} 
We only analyze the SEDs of galaxies that are either isolated systems or mergers that are experiencing coalescence (defined by a black hole separation of $<1 \mathrm{kpc}$ ) or are post-coalescence. The reason for this choice is that we only want to study systems that can be considered a single galaxy. Using this black hole separation criterion excludes early stage mergers, which would often be considered separate systems in low-redshift observations (although they would be unresolved in the FIR at high redshift when observed with single-dish FIR or submillimeter telescopes). Moreover, when the system consists of widely separated galaxies, the radiative transfer within individual galaxies is decoupled (i.e., the radiation within one galaxy does not have a significant effect on the dust in the other galaxy when the two galaxies are separated by many kiloparsecs). Using a less conservative criterion, such as $5 \mathrm{kpc}$, does not qualitatively affect our results.

\section{PREDICTING THE SEDS BASED ON PCA}

Given a sample of FIR SEDs, PCA finds the mean SED of the entire sample $\left(\left\langle\lambda L_{\lambda}\right\rangle\right)$ and based on the mean SED, finds the PCs that encapsulate most of the variance of the entire sample. PCs are eigenvectors that are orthogonal to each other. The PCs are not guaranteed to have a physical interpretation; rather, they are a tool to reduce the dimensionality of the problem. The PCs are ranked in terms of how much of the variance is explained by each PC.

Because we are interested in studying the shape of the SEDs, we normalize the FIR SEDs by dividing them by the IR luminosity. ${ }^{10}$ The units of the data on which the PCA is performed do not affect the results, and we have chosen to work in dimensionless units of $\lambda L_{\lambda} / L_{\mathrm{IR}}$.

The SED belonging to a given galaxy ( $j$ ) in the sample can be reconstructed as a linear combination of PCs:

$$
\lambda L_{\lambda, j}=\left\langle\lambda L_{\lambda}\right\rangle+\sum_{i=1}^{N} C_{i, j} \times \mathrm{PC}_{i},
$$

where $N$ is the number of PCs used, $\mathrm{PC}_{i}$ is the $i$ th $\mathrm{PC}$, and $C_{i, j}$ is the coefficient of the $i$ th $\mathrm{PC}$ for the $j$ th galaxy.

Having identified the most important PCs, we then examine how the coefficients of the PCs correlate with the various global physical parameters of the galaxies. Our goal is to be able to predict the PC coefficients, and thus the FIR SED, of a galaxy based on a small number of galaxy properties, such as the SFR. To predict the PC coefficients, we use functions of the following form:

$$
C_{i, j}^{\prime}=\alpha_{i}+\sum_{k=1}^{M} \beta_{i, k} \log P_{i, j, k}
$$

where $C_{i, j}^{\prime}$ is the predicted value of the $i$ th coefficient for galaxy $j, \alpha_{i}$ is the fit intercept for the $i$ th PC coefficient, $\beta_{i, k}$ is the fit coefficient for the $i$ th PC coefficient and $k$ th parameter, and $P_{i, j, k}$ is galaxy $j$ 's value for the $k$ th physical property used to predict the $i$ th PC coefficient; example properties include the SFR and dust mass. As discussed below, we found that two PCs were sufficient to explain $97 \%$ of the variance. Thus, we used $N=2$. To predict the PC coefficients, we tried relations with both a single physical property $(M=1)$ and a pair of physical properties $(M=2)$.

\footnotetext{
${ }^{10}$ We define $L_{\mathrm{IR}}$ as the integral of the SED over the wavelength range of $8-1000 \mu \mathrm{m}$.
}

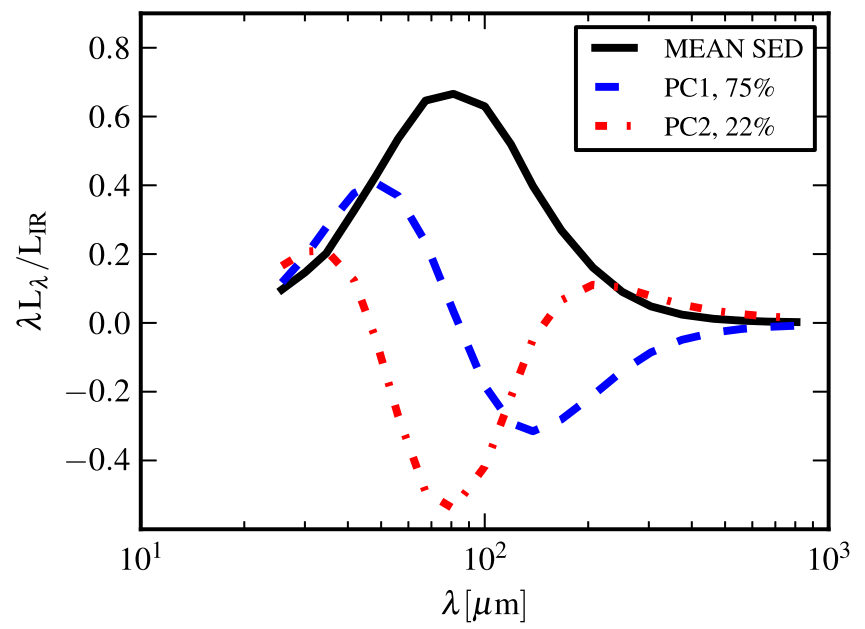

Figure 1. The black solid line corresponds to the mean FIR SED of our full sample; it has been normalized by dividing by $L_{\mathrm{IR}}$. The first (PC1; blue dashed line) and second (PC2; green dashed-dotted line) PCs are also shown. These PCs are added to the mean with a unique coefficient for each galaxy to reconstruct the individual FIR SEDs. The fraction of the variance in the data that is explained by each PC is indicated as a percentage in the legend. Together, the top two PCs can explain 97\% of the total variance of the data in the full SED sample, which includes both low- and high-redshift simulated galaxies.

Given predicted values for a galaxy's PC coefficients determined using Equation (2), we predict its SED as follows:

$$
\lambda L_{\lambda, j}^{\prime}=\left\langle\lambda L_{\lambda}\right\rangle+\sum_{i=1}^{N} C_{i, j}^{\prime} \times \mathrm{PC}_{i} .
$$

To quantify how well an SED can be predicted, we use the following quantity:

$$
\chi_{r}^{2}=\frac{1}{d f} \sum_{i=1}^{P} \frac{\left(L_{\lambda_{i}}-L_{\lambda_{i}}^{\prime}\right)^{2}}{\sigma_{\lambda_{i}}^{2}},
$$

where $L_{\lambda_{i}}$ and $L_{\lambda_{i}}^{\prime}$ denote the true and predicted luminosity density values at wavelength $\lambda_{i}, \sigma_{\lambda_{i}}$ is the uncertainty at wavelength $\lambda_{i}, P=20$ is the total number of wavelength bins in the FIR, and $d f=P-M-1$ is the number of degrees of freedom. Because our data are noise-free, for the purposes of calculating the $\chi_{r}^{2}$ value, we have arbitrarily assumed a signalto-noise ratio of 5 in each band (i.e., $\sigma_{\lambda_{i}}=0.2 L_{\lambda_{i}}$ ). ${ }^{11}$

\section{PCA RESULTS}

We performed PCA on the FIR $(\lambda>25 \mu \mathrm{m})$ SEDs from our entire sample, which includes both low- and high-redshift simulated galaxies. Figure 1 shows the mean SED and first two PCs of our sample. The percentage of the variance in the SEDs that can be explained by each PC is also indicated in the Figure. Each PC is multiplied by a coefficient (which is unique for each galaxy) and added to the mean SED of the sample to reconstruct the FIR SED of a particular galaxy. The coefficient can be negative or positive. From Figure 1, it is clear that

\footnotetext{
${ }^{11}$ We used the form of the reduced chi-squared statistic, but avoid using this terminology and do not mean to imply that the statistic should follow a chisquared distribution (because the uncertainties are arbitrary). However, we opted to use the $\chi_{r}^{2}$ notation so that the reader can easily recall the definition of the quantity. Thus, the $\chi_{r}^{2}$ values are useful in a relative sense (i.e., to determine which SEDs are predicted better than others), but the absolute values are not very meaningful.
} 

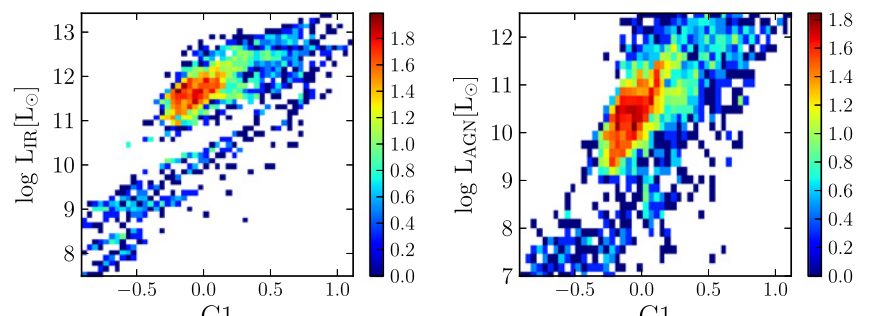

$\mathrm{C} 1$

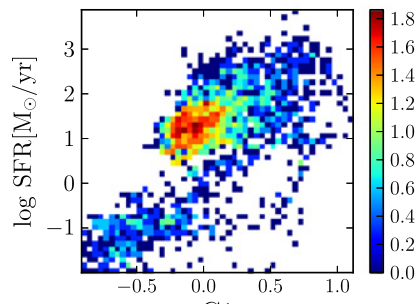

$\mathrm{C} 1$
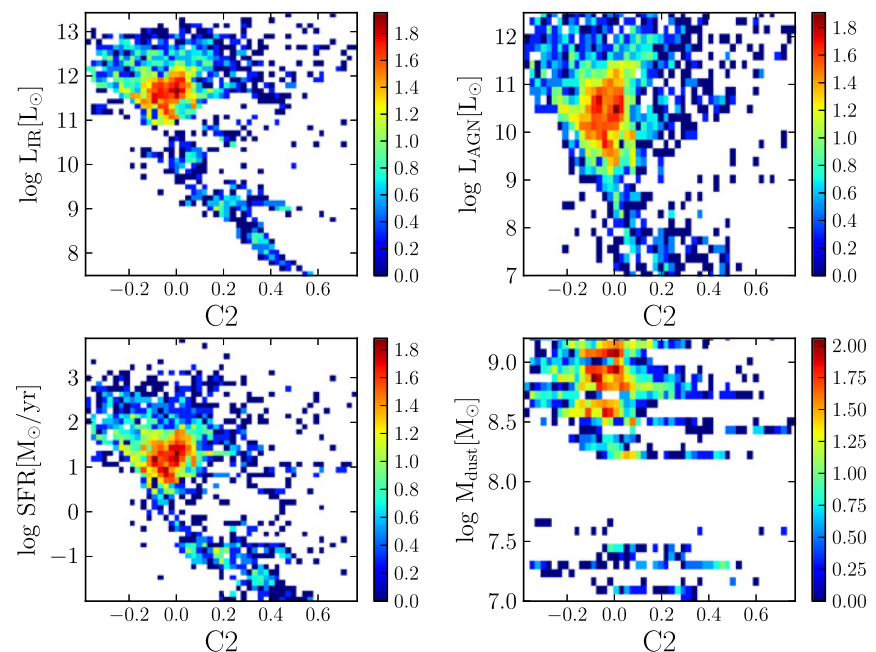

Figure 2. The top set of four figures shows how the coefficient of the first PC depends on four global physical parameters of the galaxies in the entire sample: IR luminosity (upper left), AGN luminosity (upper right), SFR (lower left), and dust mass (lower right). The bottom four figures show the same plots for $C 2$. In each panel, the parameter value is shown on the $y$-axis, and the $x$-axis corresponds to the value of the coefficient. The color of each bin corresponds to the logarithm of the number of points in the bin, as specified by the colorbars. $C 1$ is correlated with IR luminosity, SFR, and AGN luminosity, although there is a large scatter. This result indicates that as the IR luminosity, SFR, or AGN luminosity is increased, the SED peak shifts toward shorter wavelengths. $C 2$ is anti-correlated with all four physical parameters, although the correlations are weaker (see the text for details). This result indicates that there is a weak tendency for higher-luminosity or/and higher dust mass galaxies to have narrower FIR SEDs.

adding the first PC (PC1) with a positive coefficient to the mean FIR SED tends to make the SED warmer with respect to the mean SED (i.e., the wavelength at which the FIR emission peaks shifts to shorter wavelengths); if it is added with a negative coefficient, the resulting SED is cooler than the mean. Thus, the coefficient of PC1 can be considered a proxy for the effective dust temperature of the SED. In contrast, the second PC (PC2) affects the width of the SED: if it is added with a positive coefficient to the mean FIR SED, it tends to broaden the SED by increasing the power in the wings and removing power from the center. Conversely, it makes the FIR SED peak narrower if added with a negative coefficient.

The coefficients of the PCs for a galaxy determine how its FIR SED differs from the mean SED. Figure 2 shows how the coefficients of PC1 and PC2 for each galaxy in the entire sample (which we refer to as $C 1$ and $C 2$, respectively), depend on four different global physical parameters (SFR, AGNs luminosity, IR luminosity, and dust mass) that we expect to affect the FIR SEDs. We focus on these specific parameters because they are simple global parameters that are clearly important for radiative transfer, and are typically available in SAMs. The first two characterize the radiation that can potentially heat the dust. The IR luminosity then tells us how much radiation is absorbed by dust; of course, this quantity depends on both the bolometric luminosity of the stars and AGN and what fraction of the intrinsic luminosity is absorbed. $^{12}$ The dust mass characterizes the radiation sinks. As detailed below, we also explored using other individual or pairs of parameters to predict the coefficient values, but do not show them in this plot because we do not focus on them in the bulk of the analysis below.

Figure 2 indicates that $C 1$ correlates with IR luminosity (with a Pearson correlation coefficient of $r=0.71$ ), SFR $(r=0.60)$, and AGN luminosity $(r=0.73)$, although there is a large scatter. This result indicates that as the IR luminosity, SFR, or AGN luminosity is increased, the SED peak shifts toward shorter wavelengths, which is to be expected because of the known correlation between effective dust temperature and IR luminosity. The correlations between $C 2$ and the four properties shown in Figure 2 are all weak negative correlations (all have $-0.5 \lesssim r<0$ ). This result indicates that there is a weak tendency for higher-luminosity or/and higher dust mass galaxies to have narrower FIR SEDs.

Other than correlations between the PCs and the global parameters, it is also interesting to consider whether there is a correlation between the PC coefficients. Although PC1 and PC2 are orthogonal by construction, their coefficients may still be correlated. In our case, a correlation between $C 1$ and $C 2$ would indicate a relation between the effective temperature of the SED and its broadness. To see if such a correlation exists, we plot $C 1$ versus $C 2$ for all the SEDs in our sample in Figure 3. We find that $C 1$ and $C 2$ are uncorrelated.

Because the scatter in the correlations between the coefficients and global galaxy parameters shown in Figure 2 is large, it is worth considering whether we can better predict the coefficients using two parameters simultaneously. In particular, thermal equilibrium considerations suggest that combining dust mass and a luminosity-related parameter (e.g., IR luminosity or SFR) could be promising. ${ }^{13}$ Thus, in Figure 4, we show the results of predicting $C 1$ (top) and $C 2$

\footnotetext{
12 Throughout this work we use $L_{\mathrm{IR}}$ calculated by integrating the SEDs over the wavelength range of $8-1000 \mu \mathrm{m}$. When dust self-absorption is negligible, this quantity is independent of viewing angle and identical to the luminosity absorbed by dust. However, when dust self-absorption is non-negligible, $L_{\mathrm{IR}}$ can depend on viewing angle, whereas the luminosity absorbed by dust is an intrinsic property of the simulated galaxy that does not depend on viewing angle (see Hayward et al. 2011 for more details). We opt to use $L_{\mathrm{IR}}$ rather than the absorbed luminosity because (1) the former can be inferred from observations without recourse to radiative transfer modeling, (2) the average of $L_{\mathrm{IR}}$ taken over a sufficient number of viewing angles must equal the absorbed luminosity, and (3) dust self-absorption does not lead to significant variation in $L_{\mathrm{IR}}$ with viewing angle for the bulk of the simulated galaxies.

${ }^{13}$ We did not use the AGN luminosity as a predictor despite the correlation evident in Figure 2 because, for most of the simulated galaxies, the AGN contributes less than $10 \%$ of the bolometric luminosity and the FIR luminosity is not AGN dominated. (A detailed analysis of the contribution of AGNs to FIR emission will be presented in C. C. Hayward et al. 2016, in preparation.) Consequently, the correlation between the AGN luminosity and $C 1$ does not indicate causation; rather, it arises because in the simulations that we analyze, the black hole accretion rate and SFR are correlated.
} 


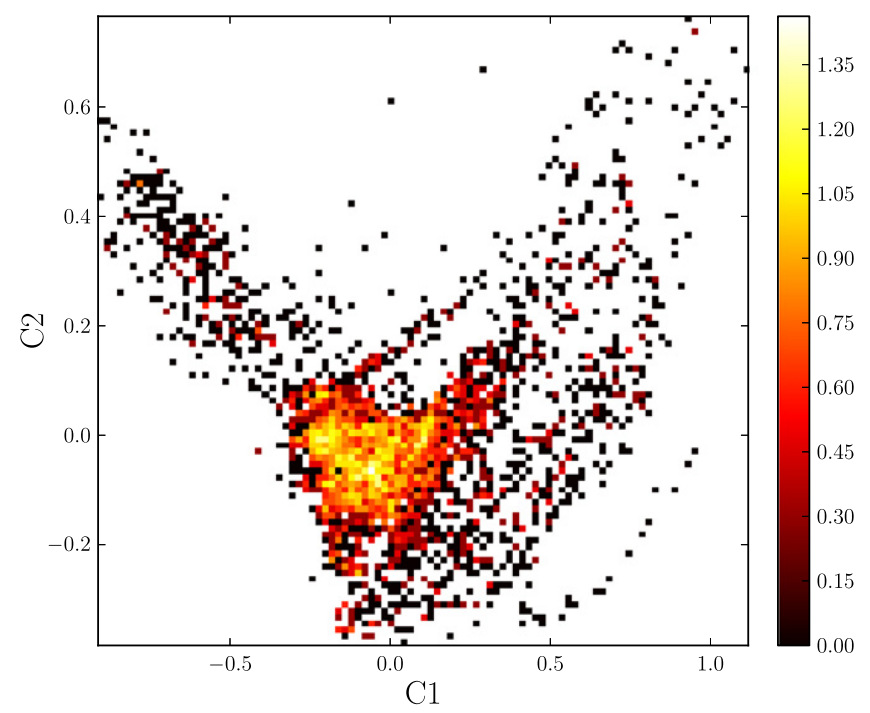

Figure 3. Biplot of $C 1$ vs. $C 2$. The color bar indicates the logarithm of the number of SEDs in the bin. Recall that higher values of $C 1$ correspond to hotter SEDs, and higher values of $C 2$ correspond to broader SEDs. The white space corresponds to no data in this plot. There appears to be a negative correlation between $C 1$ and $C 2$ at values of $C 1$ less than -0.3 . However, the density of our data points are more than 10 times higher in the dense (yellow) region where we see no correlation between $C 1$ and $C 2$. Therefore, we tend to not drive strong conclusions based on the possible correlation between $C 1$ and $C 2$ when $C 1<0$. Regions outside $-0.3<C 1<0.4$ and $-0.3<C 2<0.1$ are sparsely populated and we cannot reach a firm conclusion by studying those regions.

(bottom) in four different manners: (1) using $\log L_{\mathrm{IR}}$ (e.g., $C 1$ $=A \log L_{\mathrm{IR}}+B$ ), (2) using $\log \mathrm{SFR}$ (e.g., $C 1=A \log \mathrm{SFR}$ $+B$ ), (3) using $L_{\mathrm{IR}}$ and $M_{\text {dust }}$ (e.g., $C 1=A \log L_{\mathrm{IR}}+$ $\left.B \log M_{\text {dust }}+C\right)$ and (4) using $\log$ SFR together with $\log M_{\text {dust }}$ (e.g., $C 1=A \log$ SFR $\left.+B \log M_{\text {dust }}+C\right) .{ }^{14}$ It is clear that combining the $\log L_{\mathrm{IR}}$ and $\log M_{\text {dust }}$ results is the best way to predict $C 1$, and this combination is superior to $\log L_{\mathrm{IR}}$ alone (i.e., incorporating the dust mass reduces the error in the prediction). For $C 2$, the combination of $\log L_{\mathrm{IR}}$ and $\log M_{\text {dust }}$ is again superior to the others, but the differences in predictive power are less significant than for $C 1$. Our best-fitting relations for $C 1$ and $C 2$ are

$$
\begin{aligned}
C 1= & 0.52 \log \left(\frac{L_{\mathrm{IR}}}{L_{\odot}}\right)-0.47 \log \left(\frac{M_{\text {dust }}}{M_{\odot}}\right)-1.88 \\
C 2= & -0.014 \log \left(\frac{L_{\mathrm{IR}}}{L_{\odot}}\right)-0.10 \log \left(\frac{M_{\text {dust }}}{M_{\odot}}\right) \\
& +1.05 .
\end{aligned}
$$

We note that the coefficients for $\log L_{\mathrm{IR}}$ and $\log M_{\text {dust }}$ in Equation (5) have similar magnitudes but opposite signs. This suggests that the value of $C 1$ depends on the ratio $L_{\mathrm{IR}} / M_{\text {dust }}$. We discuss this in detail in Section 8.1. C2 depends only weakly on dust mass and is effectively independent of $L_{\mathrm{IR}}$. Thus, there is a mild tendency for sources with higher dust masses to have narrower SED peaks. Because of the weakness of the dependence, we will not interpret it further.

We now investigate how well we can predict the SEDs by predicting the PC coefficients using Equations (5) and (6), and using Equation (3) to predict the SED. Figure 5 compares the

\footnotetext{
${ }^{14}$ We have explored using various other combinations of parameters and found that these combinations had the best predictive power.
}
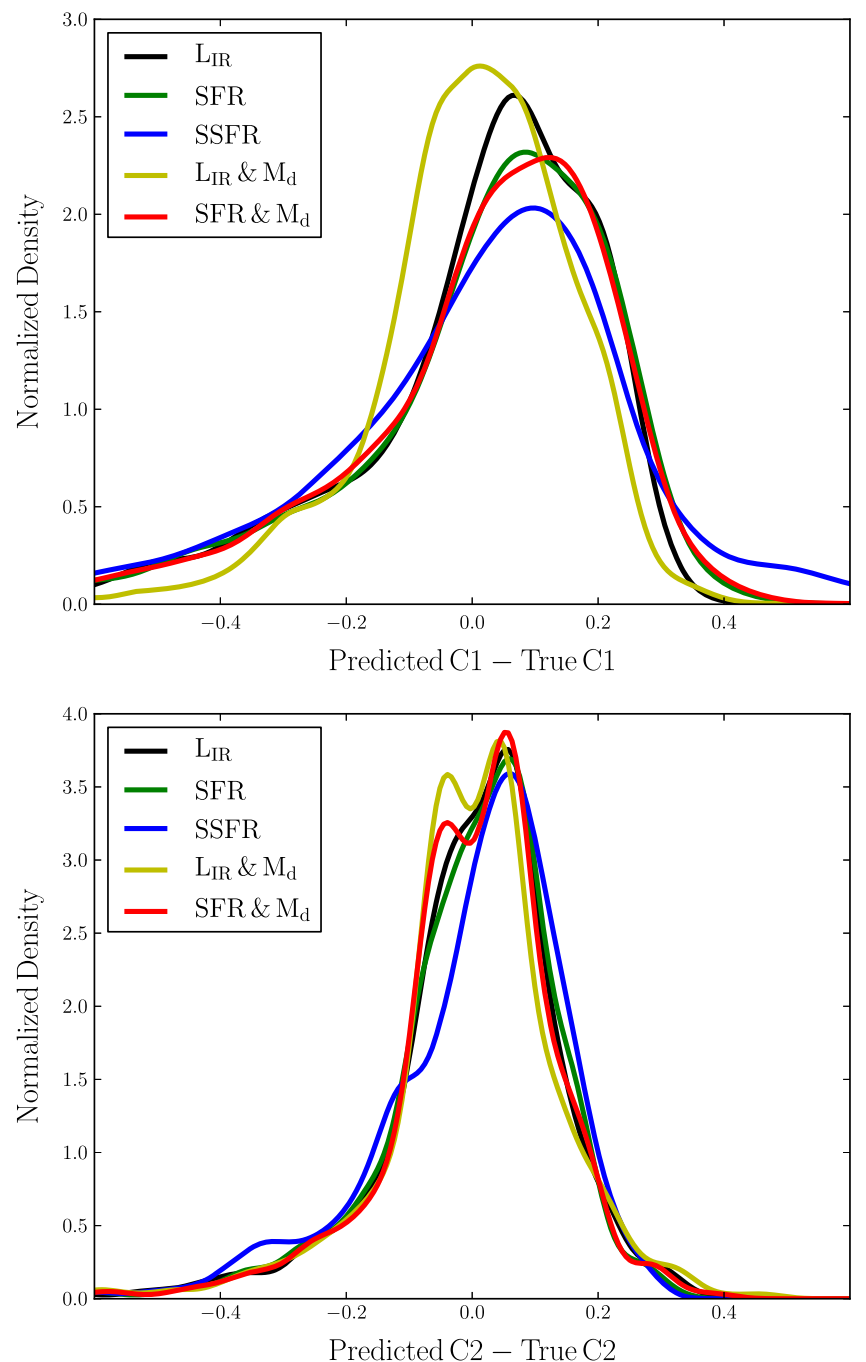

Figure 4. The top panel shows the difference between the predicted and true values of $C 1$ (the coefficient of the first PC). $C 1$ is estimated using five different estimators that depend on either one or two physical parameters; see the figure legend for the parameters used. In all cases, the logarithm of the parameter is used. Using both IR luminosity and dust mass helps reduce the bias in the predicted $C 1$ values compared with the other estimators. In the bottom panel, the same is shown for $C 2$. Again, the combination of IR luminosity and dust mass is superior to the others, although the difference in predictive power is less than for $C 1$.

predicted and true SEDs for nine randomly chosen SEDs from our entire sample. The true SED is shown in blue. The red (green) line is the predicted SED obtained by adding the first PC (first and second PC) to the mean SED with the coefficient value(s) predicted based on the galaxy's $L_{\mathrm{IR}}$ and $M_{\text {dust }}$ values (using Equations (5) and (6)). The red (green) number in each panel indicates the $\chi_{r}^{2}$ value (Equation (4)) that is obtained when only PC1 (both PC1 and PC2) is (are) used to predict the SED. From these examples, we see that the FIR SEDs are generally predicted very well near their peaks. Generally, when $\chi_{r}^{2}>1$, the reason is that the predicted and actual FIR SEDs differ at long wavelengths. Finally, using PC2 improves the prediction (in particular, at long wavelengths) in only some cases, and it can actually make the prediction worse; this is true even if we use the true values of $C 1$ and $C 2$ rather than those predicted using Equations (5) and (6). We discuss these points in more detail below. 

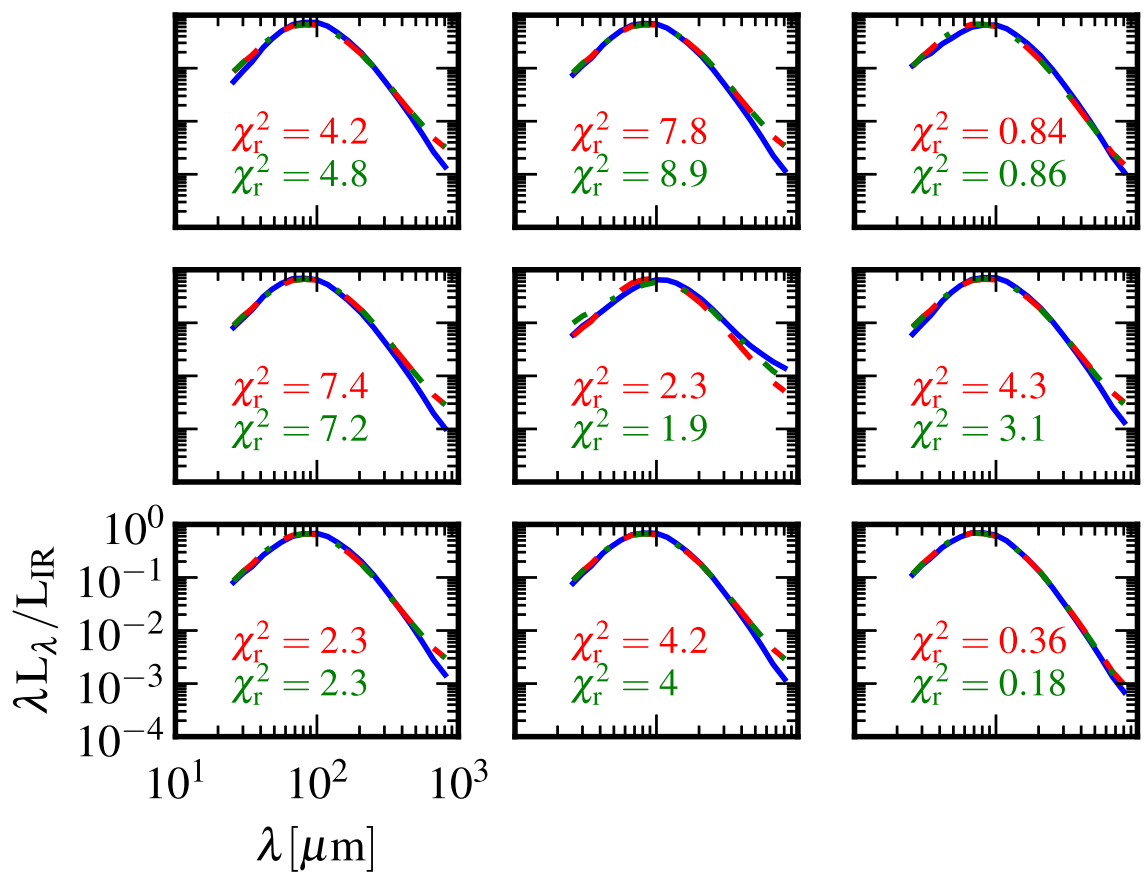

Figure 5. Comparisons of the predicted and true SEDs for nine SEDs randomly chosen from the entire sample. The blue line is the actual SED. The red (green) line is the predicted SED obtained by adding the first PC (first and second PC) to the mean SED with the coefficient value(s) predicted using $L_{\mathrm{IR}}$ and $M_{\mathrm{dust}}$. The numbers indicate the $\chi_{r}^{2}$ value (Equation (4)); a signal-to-noise ratio of five in each band was arbitrarily assumed when computing the $\chi_{r}^{2}$. Note that using the second PC leads to a better prediction in only some cases.

Figure 6 shows the distribution of $\chi_{r}^{2}$ (Equation (4)) for all SEDs in the full sample when the SEDs are predicted in this manner (the green line). We also show the $\chi_{r}^{2}$ distributions obtained when using only PC1 and predicting its coefficient, $C 1$, using either $L_{\mathrm{IR}}$ alone (the blue dashed line) or $L_{\mathrm{IR}}$ and $M_{\text {dust }}$ (the red solid line). A comparison of the solid red and blue dashed lines indicates that incorporating the dust mass results in significantly more accurate SED predictions compared with using $L_{\mathrm{IR}}$ alone. The median $\chi_{r}^{2}$ value is $0.56(0.82)$ when both $L_{\mathrm{IR}}$ and $M_{\text {dust }}$ (only $L_{\mathrm{IR}}$ is) are used to predict $C 1$. This result indicates that the SEDs should be parameterized in terms of $L_{\mathrm{IR}}$ and $M_{\text {dust }}$, not just $L_{\mathrm{IR}}$; we discuss this in detail below. The similarity of the green dashed and solid red lines indicates that using PC2 in addition to PC1 does not yield significantly better SED predictions in terms of $\chi_{r}^{2}$; in this case, the median $\chi_{r}^{2}$ is 0.54 , which is only 0.02 less than when PC1 alone is used (and both $L_{\mathrm{IR}}$ and $M_{\text {dust }}$ are used to predict $C 1$ ). This is consistent with our observation from Figure 5 that adding PC2 sometimes leads to a better prediction at long wavelengths but sometimes causes the prediction to be worse.

We consider the SED predictions with $\chi_{r}^{2} \gg 10$ to have failed catastrophically relative to those with $\chi_{r}^{2} \sim 1$. By exploring the locations of these galaxies in different parameter spaces, we determined that the catastrophic failures tend to have a high AGN contribution to the bolometric luminosity or/ and $L_{\mathrm{IR}} \gtrsim 10^{12.5} L_{\odot}$, as indicated by Figure 7. This figure shows each galaxy in the plane of $L_{\mathrm{IR}}$ and $L_{\mathrm{AGN}}$. The colors of the points indicate the $\chi_{r}^{2}$ value of the predicted SED. The top panel shows the results for the prediction based on only PC1, whereas the bottom panel shows the results for the prediction based on using both PC1 and PC2. Thus, the red circles represent galaxies for which the SED prediction fails catastrophically. It is clear that in both cases, the galaxies for

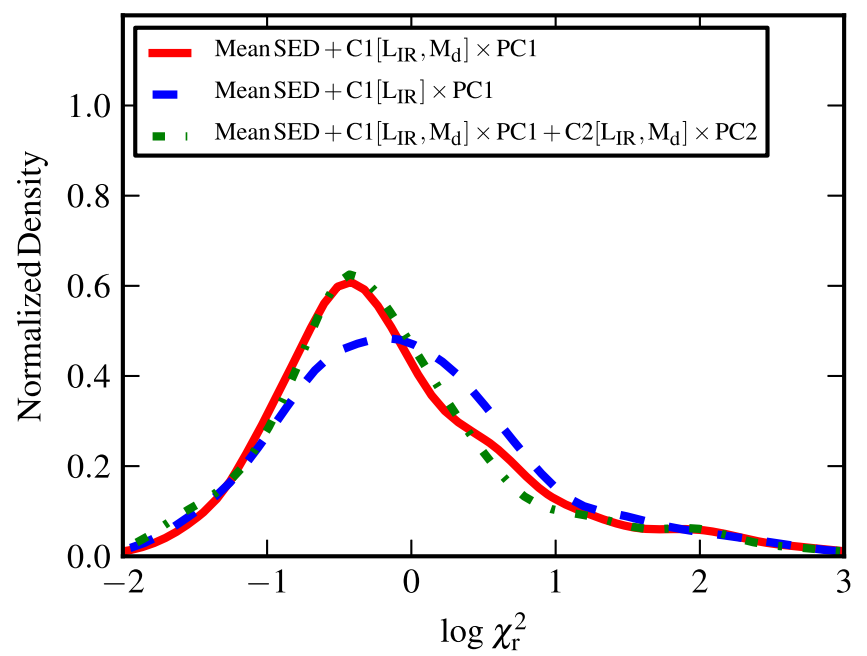

Figure 6. This figure shows how well the SEDs of the simulated galaxies can be predicted, as characterized by the $\chi_{r}^{2}$ values, using the PCs. The red line corresponds to when only PC1 is used; its coefficient is predicted using $L_{\mathrm{IR}}$ and $M_{\text {dust }}$. The dashed blue line shows the result when only PC1 is used and its coefficient is predicted using only $L_{\mathrm{IR}}$. The dotted-dashed green line shows the result when both PC1 and PC2 are used; their coefficients are predicted using both $L_{\mathrm{IR}}$ and $M_{\text {dust }}$. When $C 1$ is predicted using both $L_{\mathrm{IR}}$ and $M_{\text {dust }}$, the $\chi_{r}^{2}$ distribution shifts significantly to the left compared with when only $L_{\mathrm{IR}}$ is used; the median $\chi_{r}^{2}$ value is $0.56(0.82)$ when both $L_{\mathrm{IR}}$ and $M_{\text {dust }}$ (or only $L_{\mathrm{IR}}$ is) are used to predict $C 1$. Thus, incorporating the dust mass yields considerably better SED predictions. The similarity of the red solid and green dotted-dashed lines indicates that using PC2 does not lead to significantly better $\chi_{r}^{2}$ values, and the median value (0.54) is only slightly less than when only PC1 is used (assuming that $C 1$ is predicted using both $L_{\mathrm{IR}}$ and $M_{\text {dust }}$ ).

which the SEDs are predicted least well tend to be galaxies that have high AGN luminosities given their $L_{\mathrm{IR}}$ values (i.e., high AGN luminosity fractions) or/and have $L_{\mathrm{IR}} \gtrsim 10^{12.5} L_{\odot}$. We 

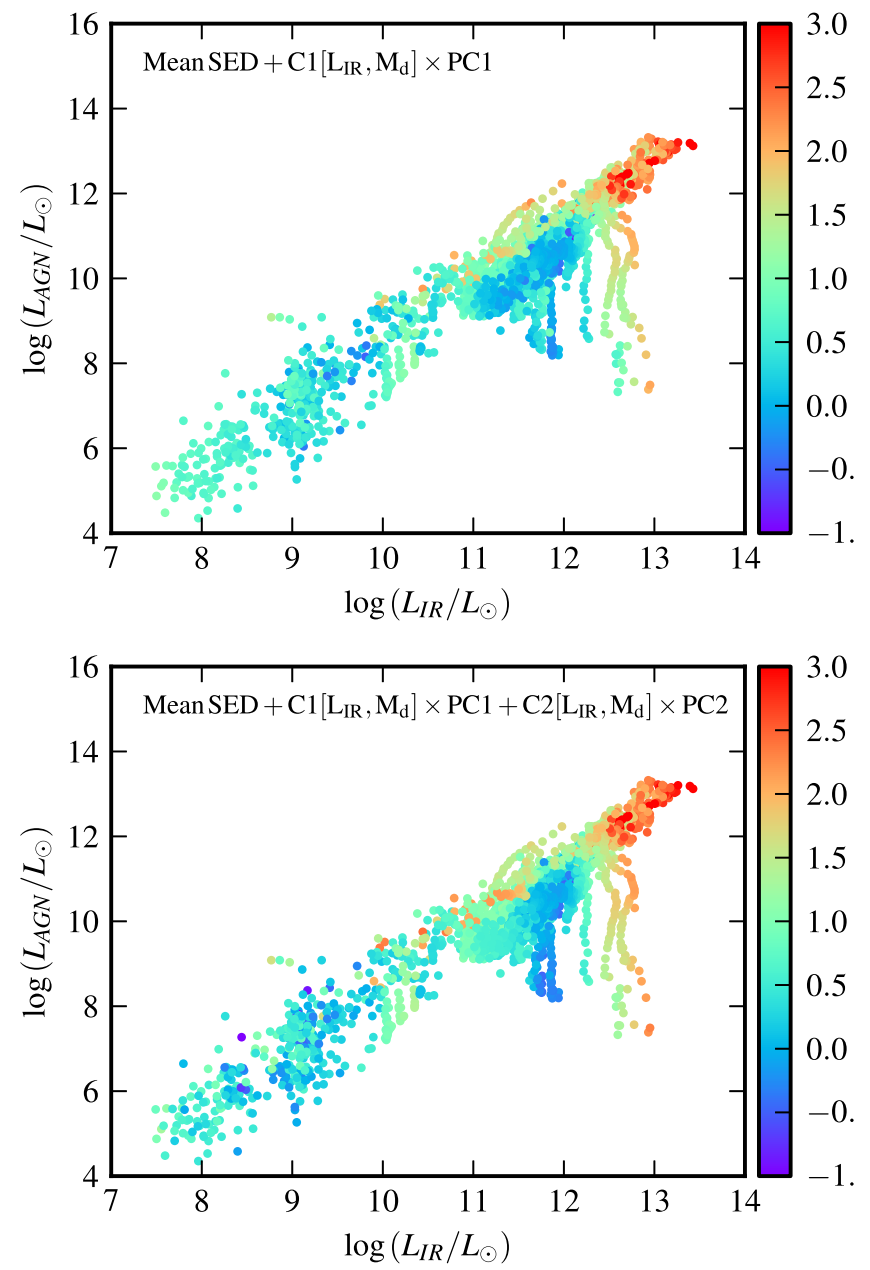

Figure 7. The $\log \chi_{r}^{2}$ values for the predicted SEDs. In the top panel, the SEDs are predicted based on using only the first PC component, with its coefficient predicted using both IR luminosity and dust mass. In the bottom panel, the SEDs are predicted based on using both PC1 and PC2; for both PCs, the coefficients are predicted using $L_{\mathrm{IR}}$ and dust mass, but the relations used differ (see Equations (5) and (6)). The colors of the circles indicate the value of the logarithm of $\chi_{r}^{2}$. The galaxies for which the SEDs are predicted least well tend to be galaxies that have high AGN luminosities given their $L_{\mathrm{IR}}$ values or/and have $L_{\mathrm{IR}} \gtrsim 10^{12.5} L_{\odot}$.

analyzed the galaxies with high AGN fractions separately, but were unable to determine global parameters that we could use to predict their SEDs well. We speculate regarding the reasons for our inability to predict the FIR SEDs of such galaxies in Section 8.4. We retain the high AGN galaxies in our subsequent analysis, but the results are not significantly changed if we exclude them because only a small fraction of the simulated galaxies have $L_{\mathrm{AGN}}>0.1$.

\section{IMPACT OF DUST MASS ON THE SEDS}

FIR SEDs are often parameterized using templates that depend on the IR luminosity alone (e.g., Chary \& Elbaz 2001; Rieke et al. 2009; Lee et al. 2013; Symeonidis et al. 2013). At fixed redshift, the effective dust temperature is observed to increase (i.e., the peak of the FIR SED shifts to shorter wavelengths) with increasing $L_{\mathrm{IR}}$ (Casey et al. 2014, and references therein). As discussed, Figure 4 indicates that using both the IR luminosity and dust mass increases our ability to predict the $C 1$ coefficients for galaxies compared with using the

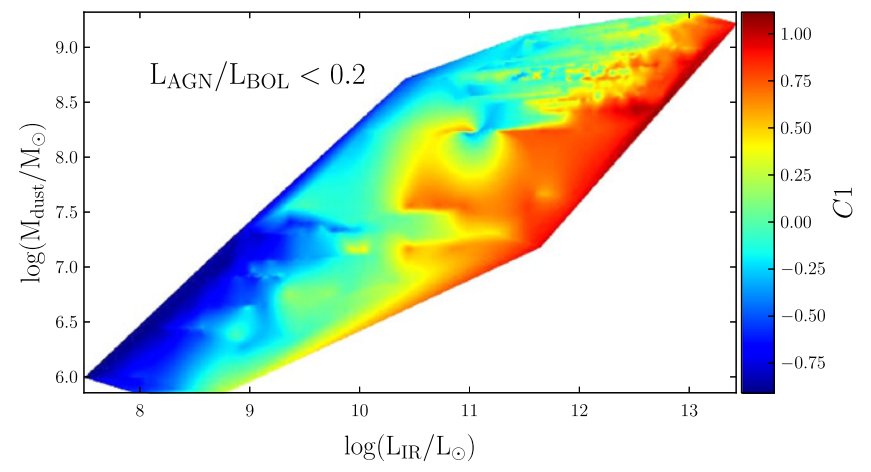

Figure 8. Shows the value of the $C 1$ coefficient (see the color bar) as a function of $L_{\mathrm{IR}}$ (on the $y$-axis) and $M_{\mathrm{dust}}$ (on the $x$-axis). The interpolation between data points is performed using the nearest-neighbor method, which can result in some artifacts, but the trend that we see is robust. At fixed $L_{\mathrm{IR}}$, higher $M_{\text {dust }}$ results in lower $C 1$ values (i.e., lower effective dust temperature). At fixed $M_{\text {dust }}$, higher $L_{\mathrm{IR}}$ results in higher $C 1$ values (i.e., higher effective dust temperature).

IR luminosity alone as a predictor. Because we know that $C 1$ basically makes an SED cooler or warmer with respect to the mean SED (higher $C 1$ values tend to make the SEDs hotter), it is instructive to examine how $C 1$ behaves on the $L_{\mathrm{IR}}$ and $M_{\text {dust }}$ plane. This is shown in Figure 8. One can see that at fixed $L_{\mathrm{IR}}$, higher values of $M_{\text {dust }}$ correspond to lower values of $C 1$ (i.e., cooler SEDs). At fixed $M_{\text {dust }}$, higher values of $L_{\mathrm{IR}}$ lead to higher values of $C 1$ (i.e., hotter SEDs).

To more explicitly demonstrate the effect of the dust mass on the SEDs, we re-ran a subset of the dust radiative transfer calculations with artificially lower and higher dust masses by changing the default dust-to-metal density ratio of $0.4-0.2$ and 0.8 , respectively. Consequently, the relative distribution of the dust remains the same, but the overall normalization and thus total dust masses are half or twice those of the standard run. Representative results for two snapshots are presented in Figure 9; each panel shows how the FIR SED for a single time and viewing angle varies as the dust-to-metal density ratio is varied. The legends specify the assumed dust-to-metal ratio and values of $L_{\mathrm{IR}}$ and $M_{\text {dust }}$ for each SED.

The results qualitatively agree with our expectations based on the above analysis. The top panel corresponds to the precoalescence phase of the most-massive major merger simulation from the $z=0$ data set. As the dust-to-metal ratio is increased, $L_{\mathrm{IR}}$ increases and the peak of the SED shifts slightly to longer wavelengths (i.e., the SED becomes colder). In this case, a substantial fraction of the luminosity is not absorbed when the dust-to-metal ratio is 0.2 . Consequently, increasing the dust-to-metal ratio leads to higher optical depths and thus a larger fraction of light is absorbed and re-emitted in the IR.

The SEDs shown in the bottom panel correspond to the M3M3e merger simulation, which is the most-massive equalmass merger simulation of $z \sim 0$ progenitors presented in Lanz et al. (2014), near the peak of the starburst induced at final coalescence. Because the luminosity is dominated by a central dust-enshrouded starburst, the simulated galaxy is already opaque to effectively all of the radiation when the dust-to-metal ratio is 0.2 . Consequently, $L_{\mathrm{IR}}$ does not increase as the dust-tometal ratio (and thus dust mass) is increased. Instead, it actually decreases by 0.1 dex; this occurs because we are considering the $L_{\mathrm{IR}}$ that is associated with a single viewing angle. When there is a non-negligible optical depth in the FIR, as can be the case for (U)LIRGs (see the discussion in Hayward et al. 2012), 

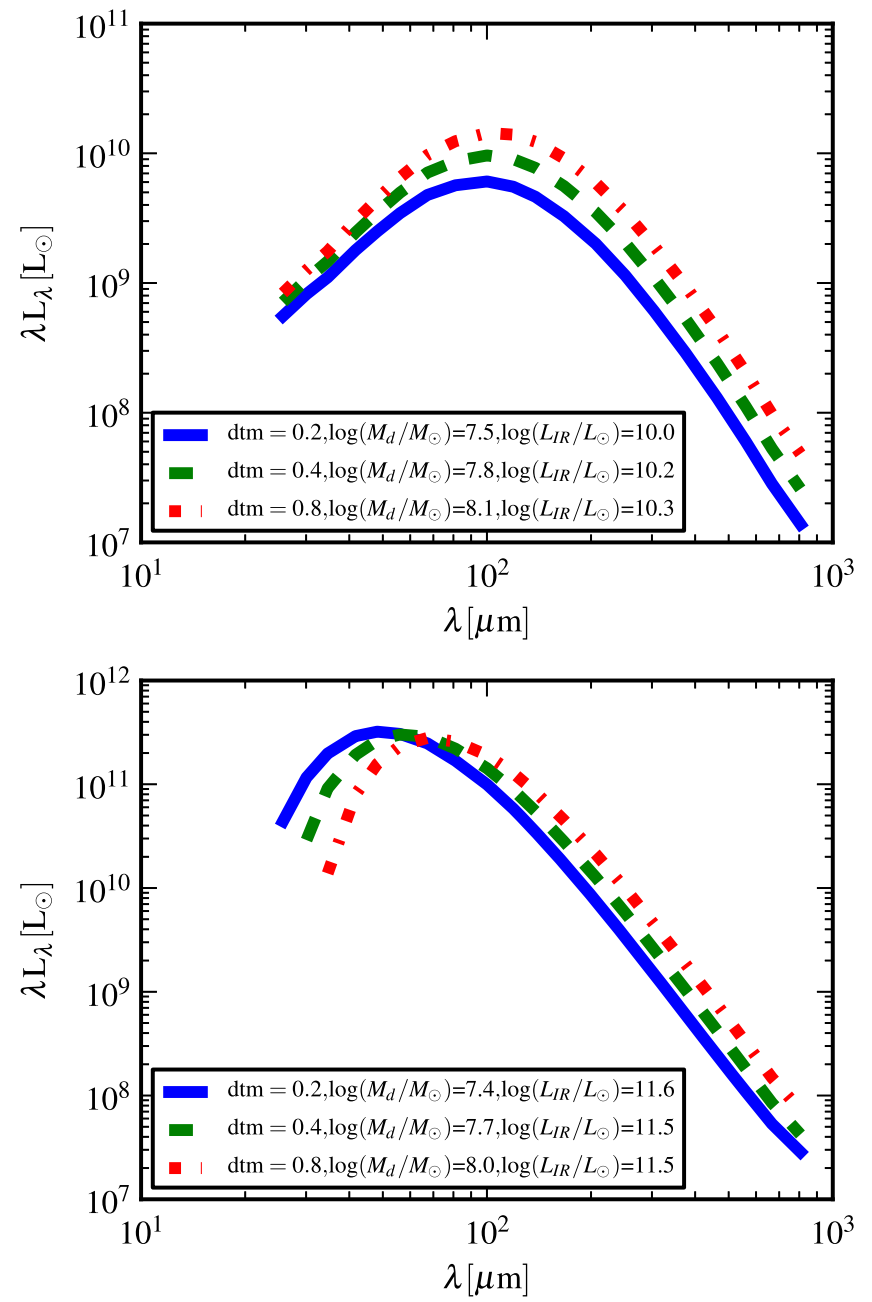

Figure 9. Shows the effect of changing the dust-to-metal ratio, and thus dust mass, on the SED. Each panel shows the SEDs of a simulated galaxy at a single time and viewed from a fixed viewing angle for three different dust-to-metal ratios, as specified in the legend; all other properties of the galaxies are unchanged. The $L_{\mathrm{IR}}$ and $M_{\text {dust }}$ values for each SED are also shown in the legend. In the top panel, the galaxy is not fully opaque when the dust-to-metal ratio is 0.2 . Consequently, as the dust-to-metal ratio is increased, $L_{\mathrm{IR}}$ increases by a factor of $\sim 2$ and the SED shifts only slightly to the right. In the bottom panel, $L_{\mathrm{IR}}$ decreases by 0.1 dex as the dust-to-metal ratio is increased; this is a consequence of the viewing-angle dependence of $L_{\mathrm{IR}}$ (see the text for details). The SED systematically shifts to longer wavelengths as the dust-to-metal ratio is increased, which is consistent with our expectation based on the relationship shown in Figure 8 and considerations of thermal equilibrium.

$L_{\mathrm{IR}}$ can depend on the viewing angle. The increased viewingangle dependence as the dust-to-metal ratio is increased explains the aforementioned decrease, because as the dust-tometal ratio is increased, more of the short-wavelength IR emission is removed from this particular line of sight. The absorbed luminosity, which is independent of viewing angle, is effectively identical in this case for all dust-to-metal ratios.

Because the $L_{\mathrm{IR}}$ values are almost identical for the three SEDs shown in the bottom panel, they provide a clean test of how the SED varies with dust mass for fixed IR luminosity. We see that, as expected based on the trend shown in Figure 8, the FIR SED systematically shifts to longer wavelengths as the dust-to-metal ratio, and thus total dust mass, is increased; all other properties of the galaxy are kept fixed.

Increasing the IR luminosity increases the temperature of the SED at fixed dust mass because there are more photons available to heat the same amount of dust; consequently, thermal equilibrium dictates that the dust temperature must also increase. Increasing the dust mass at fixed IR luminosity tends to make the SED cooler because the luminosity is distributed over a greater mass of dust (thanks to dust self-absorption). Thus, thermal equilibrium implies that the dust temperature will decrease. For an isothermal modified blackbody, the temperature scales as $T \propto\left(L_{\mathrm{IR}} / M_{\text {dust }}\right)^{1 /(4+\beta)}$, where $\beta$ is the power-law index of the dust opacity curve in the FIR (e.g., Hayward et al. 2011; Lanz et al. 2014). Although the simulated SEDs are not quantitatively well-described by this simple model (Hayward et al. 2011, 2012; Lanz et al. 2014), it does provide physical motivation for the claim that the effective dust temperature increases (decreases) when $L_{\mathrm{IR}}\left(M_{\mathrm{dust}}\right)$ is increased and $M_{\text {dust }}\left(L_{\mathrm{IR}}\right)$ is kept fixed. ${ }^{15}$ Put another way, the mean intensity "seen" by a dust grain is proportional to $L_{\mathrm{IR}} / M_{\text {dust }}$ (see Draine \& Li 2007 and Draine et al. 2007 for detailed discussions). Thus, as $L_{\mathrm{IR}}$ is increased or $M_{\text {dust }}$ is decreased, the mean intensity of light absorbed by the dust and thus typical grain temperature increases, and vice versa.

\section{IMPACT OF GALAXY SIZES ON THE SED SHAPE}

In addition to the total absorbed luminosity and dust mass, both of which must affect the SED shape because of thermal equilibrium, the geometry of radiation sources and dust can influence the SED. The surface densities of various components (e.g., all stars, young stars, gas, and dust) are global (observable) parameters that crudely characterize the global geometry of a galaxy and are often used in observational studies to interpret the evolution in the FIR SED shapes of galaxies (e.g., Elbaz et al. 2011; Rujopakarn et al. 2013). Thus, it is worthwhile to investigate whether the PCA coefficients can be predicted better by incorporating information regarding the sizes.

We calculated the 3D baryonic half-mass radii $\left(r_{e}\right)$ and used these to approximate various average volume densities by dividing integrated quantities - such as the SFR, $L_{\mathrm{IR}}$, and stellar, gas, and dust masses-by the half-mass radii cubed. ${ }^{16}$ We then investigated whether various combinations of volume densities could be used to predict the values of $C 1$ and $C 2$ better than using our standard parameterizations, $C 1\left(L_{\mathrm{IR}}, M_{\mathrm{dust}}\right)$ and $C 2\left(L_{\mathrm{IR}}, M_{\text {dust }}\right)$.

Figure 10 shows the difference between the true values of the PCA coefficients and those predicted using various estimators, as indicated in the legends (for clarity, we show only a subset of the combinations that we tried; the others fared comparably or worse). The top panel shows the results for $C 1$, and the bottom shows those for $C 2$. To relate the coefficients' values to a single galaxy parameter, we used models of the form $C 1=A \log X+B$, where $X$ is the galaxy parameter indicated in the figure legend and $A$ and $B$ are fitting coefficients. Similarly, when two parameters were used, we employed models of the form $C 1=A \log X+B \log Y+C$.

\footnotetext{
${ }^{15}$ For observational values of $L_{\mathrm{IR}}$ and $M_{\text {dust }}$ inferred from fitting galaxy SEDs with an isothermal modified blackbody, this relation is obeyed by construction. However, this is not the case for the simulations in which the SED shape, and thus effective dust temperature, can in principle depend not only on the total luminosity heating the dust and the dust mass, but also other factors, including the spatial distribution of the dust and sources and the dust composition.

${ }^{16}$ We opted to use volume densities rather than surface densities because the former are more relevant for the radiation transfer (albeit more difficult to infer from observations).
} 

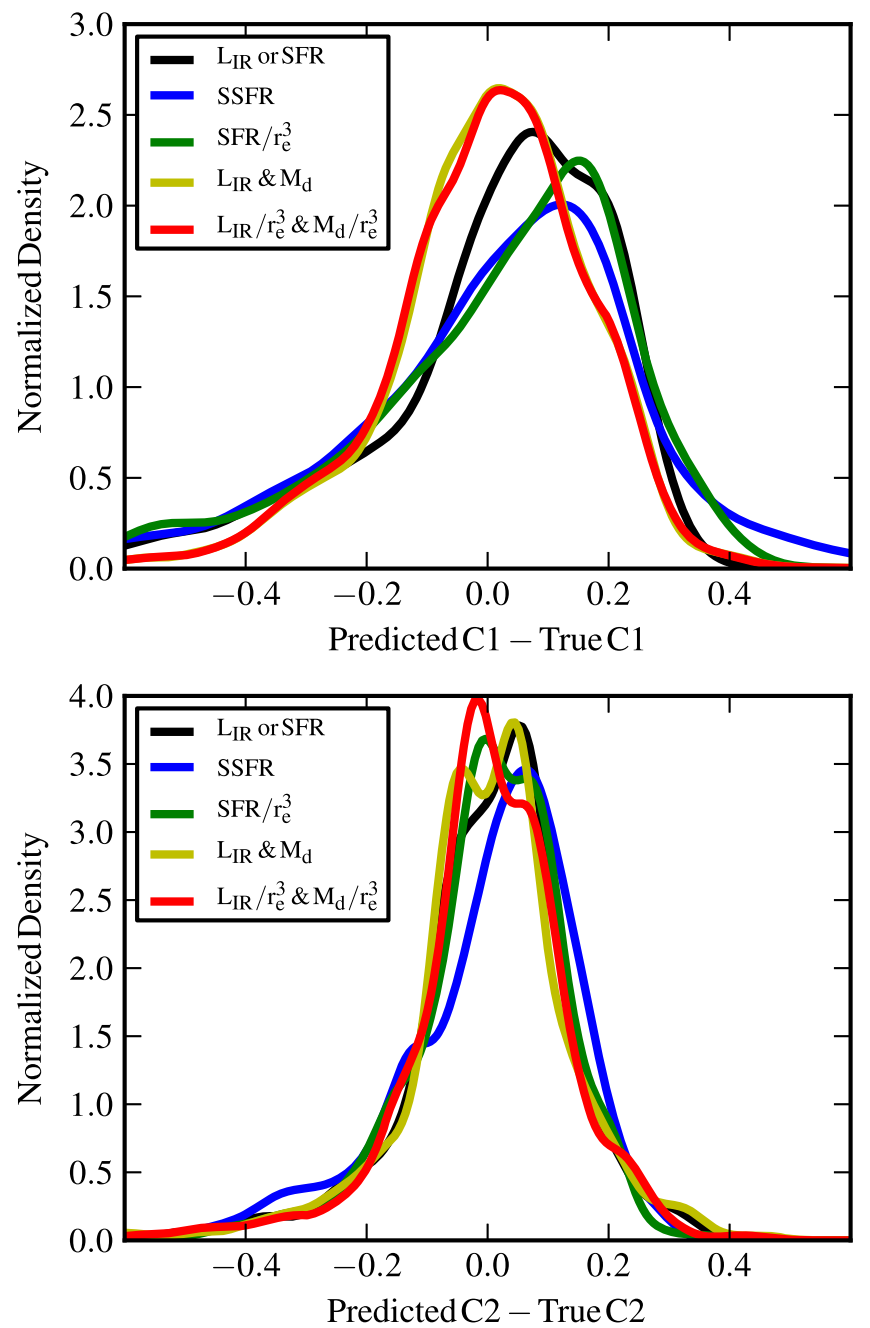

Figure 10. Difference between the predicted and true values of the coefficients for various estimators (similar to Figure 6). In this case, some of the estimators used include the galaxy sizes. The estimator used in each case is labeled in the legend, and in all cases, we use the logarithm of the parameter. For both $C 1$ (top) and $C 2$ (bottom), incorporating the size does not significantly increase the predictive power. We have experimented with other combinations of size, such as using $r_{e}^{2}$ instead of $r_{e}^{3}$, or using different measures for the radius, such as halfmass-radius of young stars, but we saw no improvement in the predictive power of the $C 1$ or $C 2$ and therefore do not present them here.

Examination of Figure 10 reveals that incorporating the size of the system when predicting the coefficients yields at best a very marginal improvement in the predictive power compared with using the total IR luminosity and dust mass values alone. The difference in the final likelihood of the model where we use only $L_{\mathrm{IR}}$ and $M_{d}$ to predict $C 1$ and consequently reconstruct the SEDs and that of the model where we use $M_{d} / r_{e}{ }^{3}$ and $L_{\mathrm{IR}} / r_{e}{ }^{3}$ to estimate the $C 1$ coefficient and consequently predict the SEDs is negligible $(\sim 0)$. Therefore, based on the Akaike Information Criterion (AIC; Akaike 1974), we prefer the model that does not have size as an extra parameter. Thus, we have not used the sizes to predict the SEDs. This lack of improvement from incorporating the galaxy size suggests that, at least for the simulated galaxies, the overall spatial extent does not play a significant role in determining the shape of the FIR SED. We discuss this perhaps surprising result in detail in Section 8.3.

\section{TWO-PARAMETER FIR SED TEMPLATES}

Motivated by these results, we now introduce a set of templates that is a function of both $L_{\mathrm{IR}}$ and $M_{\text {dust. }}{ }^{17} \mathrm{We}$ separated the simulations' FIR SEDs into $\left(L_{\mathrm{IR}}, M_{\text {dust }}\right)$ bins and calculated the median SED in each bin; these are shown in Figure 11. Each panel shows the median FIR SED for each bin as a solid line, and the 16-84th percentile range is indicated by the shaded area. The dashed lines represent the SEDs predicted by adding PC1 to the mean SED, with the coefficient value predicted by inputting the median $L_{\mathrm{IR}}$ and $M_{\text {dust }}$ values for each bin into Equation (5). The dotted-dashed lines indicate the SEDs predicted when both PC1 and PC2 are used. The SEDs have been normalized by dividing by $L_{\mathrm{IR}}$ and are colored according to the wavelength at which the FIR peak is located, with redder colors indicating longer wavelengths. The trends revealed in the aforementioned analysis are apparent from the templates: (1) at fixed $M_{d}$, the SEDs become warmer as $L_{\mathrm{IR}}$ increases (i.e., from left to right), and (2) at fixed $L_{\mathrm{IR}}$, the SEDs become cooler when $M_{\text {dust }}$ increases (i.e., from bottom to top).

Figure 12 shows the ratios of the predicted SEDs to the true SEDs, $L_{\lambda}^{\prime} / L_{\lambda}$, both when only PC1 is used (solid lines) and when both PC1 and PC2 are used (dashed lines). This figure demonstrates that the median SEDs in each bin are best predicted near their peaks. Shortward of the SED peaks, the median SEDs of most bins can be predicted to within a few tens of percent. However, for some bins the prediction is worse; in one (the bin with median values $M_{\text {dust }}=10^{6.4} M_{\odot}$ and $L_{\mathrm{IR}}=10^{8.3} L_{\odot}$ ), the SED is underpredicted by more than an order of magnitude at $\lambda \sim 35 \mu \mathrm{m}$. At wavelengths $\gtrsim 150 \mu \mathrm{m}$, the SEDs can be predicted to within a few tens of percent. However, in many bins, the SEDs are underpredicted considerably. Using PC2 in addition to $\mathrm{PC} 1$ sometimes makes the prediction better (i.e., the dashed line is closer to one than is the solid line).

The goal of PCA is to explain the variance in a data set. Thus, it is unsurprising that the peaks of the SEDs are predicted best, because this is the region that dominates the variance in the data set. Because the luminosity density at long wavelengths is $\sim 1-2$ orders of magnitude less than that at the SED peak, the long-wavelength regions of the SEDs contribute relatively little to the variance. Consequently, it would be necessary to use higher-order PCs to predict the longwavelength emission well.

\section{DISCUSSION}

\subsection{The Simplicity of the FIR SEDs of Galaxies}

We have found that the FIR SEDs of our simulated galaxies can be well predicted based on the galaxies' IR luminosities and dust masses alone. Our results extend those of Hayward et al. (2011), who demonstrated that the observed-frame submm flux densities of simulated $z \sim 2$ SMGs could be well predicted using these two parameters. Moreover, as noted in Section 4, $C 1$ effectively depends on $\log L_{\mathrm{IR}} / M_{\text {dust }}$ because the best-fitting coefficients for $\left(L_{\mathrm{IR}}\right.$ and $\left.M_{\text {dust }}\right)$ have almost the same magnitude but opposite signs $(0.52$ and -0.47 , respectively). Interestingly, in the simple isothermal modified blackbody model, the dust temperature scales with $L_{\mathrm{IR}} / M_{\text {dust }} \cdot{ }^{18}$

\footnotetext{
17 The templates are available at http://dx.doi.org/10.7910/DVN/X1OVSU.

18 However, it is important to note that how the effective dust temperatures of the simulated galaxies' SEDs depend on $L_{\mathrm{IR}} / M_{\mathrm{dust}}$ is not fully captured by this model (Lanz et al. 2014).
} 


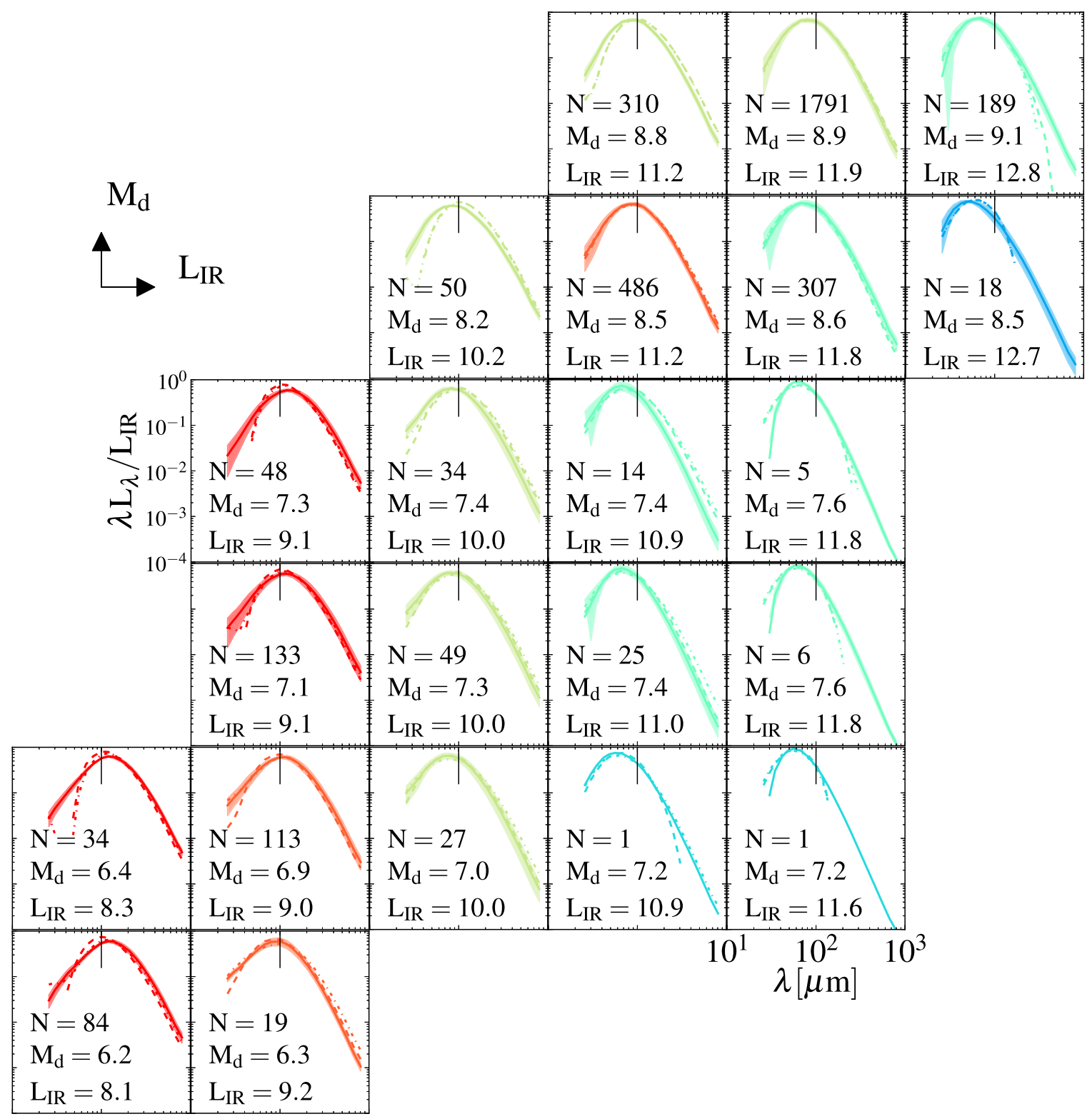

Figure 11. SEDs of our simulated galaxies binned according to IR luminosity and dust mass. In each panel, the median FIR SED in that bin is indicated by the solid line, and the shaded region represents the 16-84th percentile range of the FIR SEDs in that bin. The dashed (dotted-dashed) line shows the SED predicted using PC1 (PC1 and PC2), with the coefficient(s) predicted using the median $L_{\mathrm{IR}}$ and $M_{\text {dust }}$ values for that bin. The SEDs have been normalized by dividing by $L_{\mathrm{IR}}$. The color coding is based on where the peak of the FIR SED is located, with redder colors corresponding to longer peak wavelengths (i.e., colder effective dust temperatures). The logarithms of the median dust mass and IR luminosity (in solar units) are indicated in each panel, and the number of SEDs in each bin ( $N$ ) is also shown. The dust mass increases in the upward direction, and $L_{\mathrm{IR}}$ increases to the right. In most columns, the SEDs become redder from bottom to top; this visually illustrates the trend for increasing $M_{\text {dust }}$ to result in cooler SEDs when $L_{\mathrm{IR}}$ is fixed. In a given row, the SEDs become bluer from left to right because at fixed $M_{\text {dust }}$ the SEDs become hotter as $L_{\mathrm{IR}}$ is increased. The trend with $L_{\mathrm{IR}}$ is more apparent than with $M_{\mathrm{dust}}$ because, except for the bottom row, the $L_{\mathrm{IR}}$ values in a given row span $2-3$ orders of magnitude, whereas the $M_{\text {dust }}$ values in a given column span only 1-2 orders of magnitude. Generally, the SEDs are predicted very well near their peaks. In some cases, the SEDs are significantly underpredicted at long wavelengths, although use of PC2 reduces the amount by which the SED is underpredicted, compared with using only PC1.

In the more realistic case of continuum of dust temperatures, the mean intensity of the radiation absorbed by the dust is proportional to $L_{\mathrm{IR}} / M_{\text {dust }}$ (Draine \& Li 2007). Thus, both simple models and our simulations suggest that the ratio $L_{\mathrm{IR}} / M_{\text {dust }}$ is a key determinant of the FIR SED of a galaxy.

These results indicate that the FIR SEDs of our simulated galaxies are rather simple. The skeptical reader may suggest that this simplicity is a consequence of the simplicity of our simulations. However, even in the simulations, the FIR SEDs could potentially exhibit greater complexity because the simulated galaxies contain dust with a continuum of temperatures (which are set by the 3D interstellar radiation field, 3D distribution of dust, and grain properties). Thus, the fact that $L_{\mathrm{IR}} / M_{\mathrm{dust}}$ encodes so much of the variance in the simulated SEDs is somewhat surprising.

Similar conclusions have been obtained based on observed FIR SEDs of galaxies. In particular, by fitting observed galaxy SEDs using the model of Draine \& Li (2007), Magdis et al. (2012) argued that the redshift evolution of "main sequence" galaxies' SEDs is driven by redshift evolution in the $L_{\mathrm{IR}} / M_{\text {dust }}$ ratio. For this reason, they suggest using SED templates for the "main-sequence" galaxies that depend on $L_{\mathrm{IR}} / M_{\text {dust }}$. 


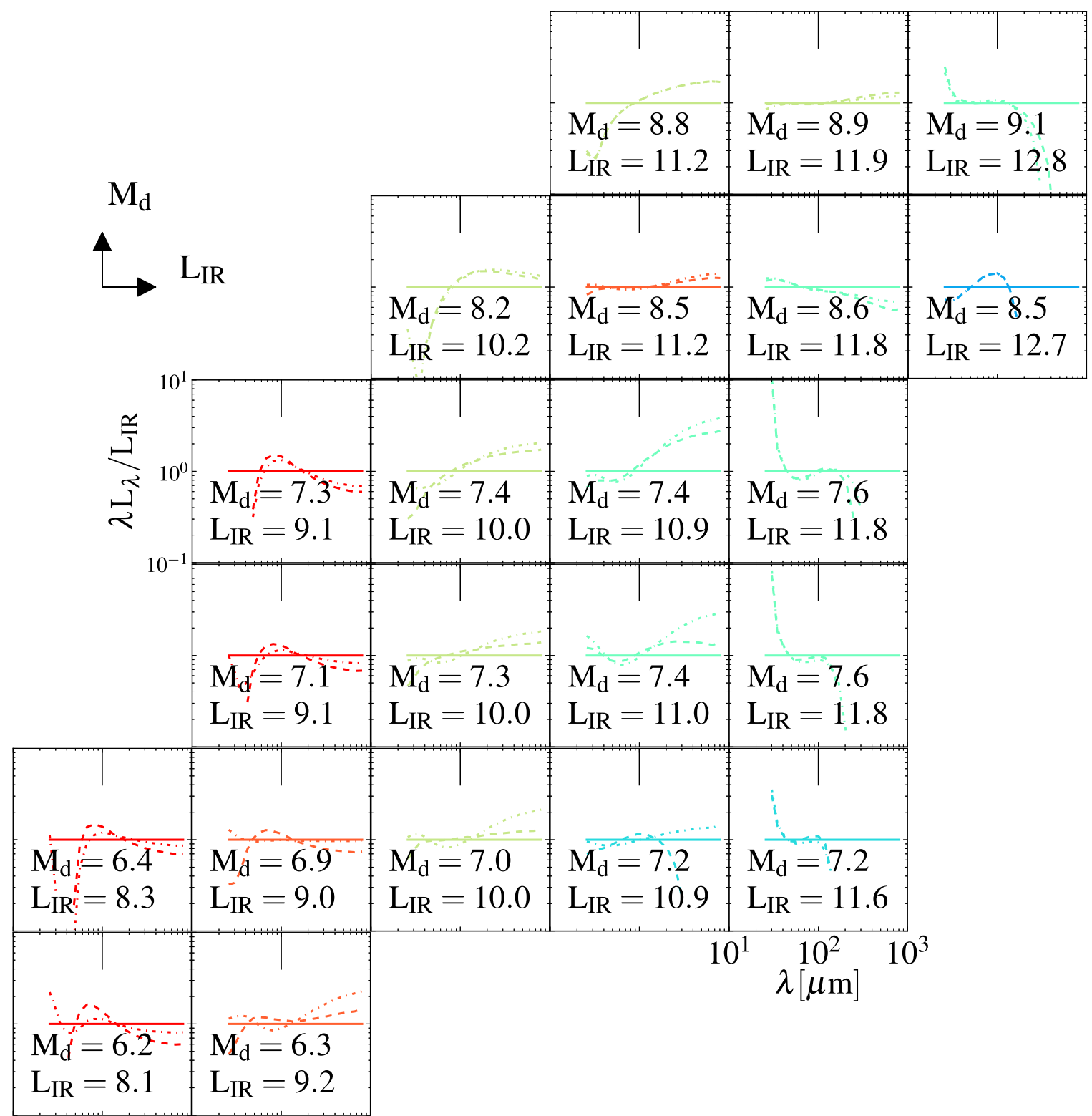

Figure 12. Predicted FIR SED divided by the true FIR SED when PC1 (PC1 and PC2) is (are) used to predict the median SED in each bin is shown by the dashed (dotted-dashed) lines. The solid lines correspond to a ratio of unity. The $\left(L_{\mathrm{IR}}, M_{\mathrm{dust}}\right)$ bins are the same as in Figure 11 . This figure clearly shows that near their peaks, the median SEDs can be predicted well based on their $L_{\mathrm{IR}}$ and $M_{\mathrm{dust}}$ values alone. At wavelengths significantly shorter or longer than the peak wavelength, the SEDs are predicted very well in some bins but can be incorrect by greater than an order of magnitude. Incorporating PC 2 makes the predictions more accurate in some cases, but it can also make them worse.

Magnelli et al. (2014) studied how the effective temperature of galaxies depends on their position in the SFR $-M_{\star}$ plane and redshift. They found that at all redshifts, the effective dust temperature smoothly increases with $L_{\mathrm{IR}}$, specific SFR, and the distance from the "main sequence" (i.e., excess SSFR relative to what one would expect for a "main sequence" galaxy of the same mass), with the latter two correlations being more significant than the first. They interpret the dependence on the distance from the "main sequence" in terms of changes in the global star formation efficiency, SFR/ $M_{\text {gas }}$. However, we note that for an approximately constant dust ratio, this quantity would serve as a proxy for $L_{\mathrm{IR}} / M_{\text {dust }}$. Thus, the results of Magnelli et al. (2014) are likely consistent with those of Magdis et al. (2012) and this work.

\subsection{Observational Support for the Importance of Dust Mass}

Observational studies of the dust masses of different types of galaxies-including submillimeter galaxies (Rowlands et al. 2014), normal massive star-forming galaxies (Dunne et al. 2011), early type galaxies (Martini et al. 2013), and highredshift galaxies (Michaowski 2015) —all point to the presence of unexpectedly large quantities of dust in high-redshift galaxies. For example, Rowlands et al. (2014) found that the dust masses of submillimeter galaxies could be as much as two orders of magnitude greater than the expectations from the inferred star-formation histories and theoretical dust production and destruction rates.

Moreover, observations indicate that at fixed $L_{\mathrm{IR}}, z \sim 2-3$ galaxies have lower effective dust temperatures than do local 


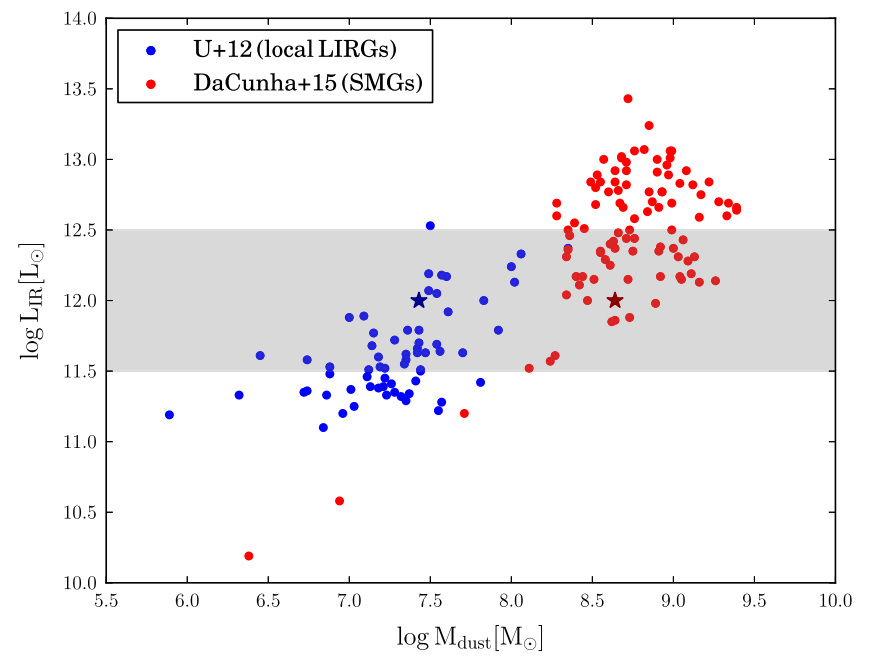

Figure 13. IR luminosity vs. dust mass for two samples of IR-luminous galaxies, local LIRGs and ULIRGs from Vivian et al. (2012), and $z \sim 2-3$ SMGs from da Cunha et al. (2015). The stars indicate the median dust mass for the subset of each sample within the shaded region, which is defined by $10^{11.5}<L_{\mathrm{IR}} / L_{\odot}<10^{12.5}$. The median dust mass of the SMGs is an order of magnitude greater than that of the local (U)LIRGs.

galaxies (Casey et al. 2014 and references therein). Our results suggest that the observed trend could be a natural consequence of high-redshift galaxies having more dust per unit IR luminosity. Thus, the evolution of the effective dust temperature-IR luminosity relation might be a consequence of evolution in the global properties of the ISM of galaxies, rather than changes in the small-scale geometry of star-forming regions.

The dust mass of a galaxy depends on the gas mass, gasphase metallicity, and dust-to-metal ratio. As stars are formed, the ISM is enriched, which can increase the dust mass. However, star formation simultaneously reduces the dust content of the ISM because the stars are formed from dustenriched gas. Simple models that encapsulate this competition between gas enrichment and consumption indicate that the maximum dust mass of a galaxy depends weakly on the gas fraction: it is maximal when the gas fraction is $37 \%$, but it varies by less than a factor of three for gas fractions in the range of 4\%-86\% (Edmunds \& Eales 1998). This result is for a closed box, but the limit also holds if outflows or unenriched inflows are allowed. Thus, although $z \sim 2-3$ ultraluminous IR galaxies (ULIRGs) are less metal-rich than local ULIRGs, they may still have higher dust masses.

There is some observational evidence that supports our claim that the lower effective dust temperatures of $z \sim 2-3$ ULIRGs are associated with higher dust masses relative to local ULIRGs. Figure 13 shows $L_{\mathrm{IR}}$ versus $M_{\text {dust }}$ for a sample of local LIRGs and ULIRGs (Vivian et al. 2012) and a sample of $z \sim 2-3 \quad$ SMGs (da Cunha et al. 2015). For $L_{\mathrm{IR}} \sim 10^{11.5-12.5} L_{\odot}$, the local ULIRGs have a median dust mass of $10^{7.43} M_{\odot}$, whereas the $z \sim 2-3$ SMGs have a median $M_{\text {dust }} \approx 10^{8.64} M_{\odot}$. Thus, the SMGs have dust masses that are more than an order of magnitude greater than those of the $z \sim 0$ ULIRGs. We argue that the increased dust masses are the reason that the SMGs have cooler SEDs. This is consistent with the suggestion from Magdis et al. (2012) that $z \sim 0.5-2$ "main sequence" ULIRGs have cooler SEDs than local ULIRGs because the former have lower $L_{\mathrm{IR}} / M_{\text {dust }}$ ratios.

We used the $L_{\mathrm{IR}}$ and $M_{\text {dust }}$ data from da Cunha et al. (2015) because they were inferred from high-resolution ALMA data and Herschel data that were deblended based on the ALMA data. Thus, their photometry should be much less affected by blending than typical data sets from Herschel and other singledish FIR/(sub)millimeter telescopes. This is highly desirable, because the fact that blending becomes more severe at longer wavelengths could cause the SEDs extracted from blended data to be colder than the true SEDs of individual sources. However, the significant caveat regarding our use of this data set is that the SMG selection is biased toward colder effective dust temperatures, and thus the $L_{\mathrm{IR}} / M_{\text {dust }}$ values of $z \sim 2$ SMGs likely do not represent the full range of $L_{\mathrm{IR}} / M_{\text {dust }}$ values exhibited by $z \sim 2$ ULIRGs. To conclusively determine how the $L_{\mathrm{IR}} / M_{\text {dust }}$ ratios of $z \sim 2$ ULIRGs compare with those of local ULIRGs, high-resolution FIR and (sub)millimeter observations of a sufficiently large, unbiased (in terms of effective dust temperature) sample of $z \sim 2$ ULIRGs are required.

\subsection{The Unimportance of Galaxy Sizes in Determining the SED Shape}

In Section 6, we demonstrated that incorporating information regarding the galaxy sizes did not significantly improve our ability to predict the FIR SEDs. This result may be surprising to some readers, given that it is often suggested that $z \sim 2$ ULIRGs have lower effective dust temperatures than local ULIRGs because the former are more extended (e.g., Elbaz et al. 2011; Rujopakarn et al. 2011, 2013). It is true that increasing the spatial extent of the absorbing material for a central source surrounded by dust (i.e., the "shell" geometry of Misselt et al. 2001) will result in colder dust because the dust grains receive a more "diluted" radiation field. This geometry may be a reasonable approximation (especially if the shell is allowed to be clumpy) for individual $\mathrm{H}$ II regions and highly obscured AGNs; indeed, more compact $\mathrm{H}_{\text {II }}$ regions exhibit hotter dust temperatures (e.g., Groves et al. 2008). However, the overall geometry of both real galaxies and our simulated galaxies is likely more similar to the "dusty" geometry of Misselt et al. (2001), in which the stars and dust are mixed, because both the SFR density and dust density are correlated with the gas density (see also Jonsson et al. 2006). In such a geometry, the temperature of the dust is insensitive to the size (assuming that the sizes of the stellar and dust distributions are scaled in the same manner; Misselt et al. 2001).

It is worth considering suggestions in the literature that the correlation between the $L_{\mathrm{IR}}$ surface density $\left(\Sigma_{L_{\mathrm{IR}}}\right)$ and $T_{d}$ is tighter than that between $L_{\mathrm{IR}}$ and $T_{d}$, which would suggest that size is important for determining the FIR SED shape, in contrast with our findings. As discussed later, the observational evidence in favor of a correlation of dust temperature with galaxy size is somewhat tentative. This seems to have become conventional wisdom, perhaps based more on the the intuition that an individual dust grain will become cooler as it moves away from the radiation source. However, this naive physical picture is overly simplistic: as already demonstrated by Misselt et al. (2001) more than a decade ago, the overall size of the galaxy does not affect the characteristic dust temperature in a more realistic geometry in which dust and sources are intermixed. 
A literature search did reveal a few works that are particularly relevant. Chanial et al. (2007) used radio continuum-derived sizes as a proxy for IR sizes to compute the IR luminosity surface density for two samples of IRluminous galaxies: one selected at $60 \mu \mathrm{m}$ and the other selected at $100 \mu \mathrm{m}$. They argued that introducing galaxy size as an additional parameter reduced the dispersion in the $L_{\mathrm{IR}}-T_{d}$ relation. Moreover, their bootstrap estimation of the power-law indices indicated that the $60-100 \mu \mathrm{m}$ flux ratio (which was used as a proxy for dust temperature) depended on the IR surface density. However, they did not investigate the effect of including dust mass as a parameter. Thus, their results are not necessarily inconsistent with our result that incorporating size does not increase our ability to predict the FIR SEDs relative to when $L_{\mathrm{IR}}$ and $M_{\text {dust }}$ are used. It would certainly to be worthwhile to perform a similar analysis using Herschel data and including dust mass as a parameter.

Elbaz et al. (2011) found that IR8 $\equiv L_{8 \mu \mathrm{m}} / L_{\mathrm{IR}}$ and $\Sigma_{L_{\mathrm{IR}}}$ are correlated. They argued that IR8 is correlated with the IR luminosity surface density (i.e., compactness) and "starburstiness" of galaxies. (However, we note that it was not quantitatively demonstrated whether the strength of this correlation differed significantly from that between IR8 and $L_{\mathrm{IR}}$.) They then constructed template SEDs for extended ("main sequence") and compact ("starburst") galaxies. It could be argued that the differences in the effective dust temperatures of their "starburst" and "main sequence" SEDs (40 and $31 \mathrm{~K}$, respectively) supports the suggestion that effective dust temperature is inversely correlated with IR luminosity surface density. However, we note that their compact galaxies, which they suggest are starbursts, are systematically more luminous than the non-compact, "main sequence" galaxies (e.g., their Figure 16). Thus, the differences in the two SEDs may be driven by differences in IR luminosity, and it is unclear whether galaxy size plays a role.

Rujopakarn et al. (2011) reported a correlation between $L_{\mathrm{IR}}$ and $\Sigma_{L_{\mathrm{IR}}}$; as in Chanial et al. (2007), the IR size was inferred from radio continuum observations. However, the authors did not quantify the extent to which using IR luminosity surface density rather than IR luminosity reduced the dispersion in the correlations (or if it even did). Closer inspection of the sizes in Rujopakarn et al. (2011) indicates that they exhibit a large scatter with no clear trend between size and $L_{\mathrm{IR}}$, except for local LIRGs, which have smaller sizes than less-luminous local star-forming galaxies. Therefore, the correlation between $L_{\mathrm{IR}}$ and $\Sigma_{L_{\mathrm{IR}}}$ in Rujopakarn et al. (2011) is likely to be largely driven by the intrinsic correlation between $L_{\mathrm{IR}}$ and $L_{\mathrm{IR}}$. Moreover, in an independent study, Tateuchi et al. (2015) measured the $\mathrm{Pa} \alpha$ sizes of local LIRGs and found no correlation between $L_{\mathrm{IR}}$ and size.

We conclude that there is weak existing evidence that the correlation between the effective dust temperature and IR luminosity density is stronger than that between effective dust temperature and IR luminosity (i.e., that galaxy size affects the IR SED shape when $L_{\mathrm{IR}}$ is fixed), and this topic is worthy of further investigation. ALMA should be incredibly helpful in this regard because it can probe the rest-frame FIR emission of high-redshift galaxies at sub-arcsecond resolution. Thus, ALMA can be used to directly measure FIR sizes rather than relying on sizes from radio observations (although nuclear starbursts may still be unresolved; Scoville et al. 2015). Moreover, ALMA maps can be used to de-blend data from FIR telescopes such as Herschel, thereby providing FIR SEDs that are minimally affected by blending. We look forward to seeing whether ALMA observations confirm our predictions.

Owing to computational constraints, we only computed integrated SEDs, not the luminosity surface densities. Our analysis indicates that the 3D size, which is arguably the most physical measure of the relative distribution of stars and dusts, plays a subdominant role in the determination of the FIR SED shape. However, it is possible that a correlation between luminosity surface density at some wavelengths arises because of radiative transfer effects. For example, all else being equal, if the total dust mass is increased by increasing the dust-to-metal ratio (such that the relative spatial distribution is fixed), dust self-absorption can cause the radius measured from dust emission to increase because the $\tau=1$ surface will move outward. Thus, the luminosity surface density would decrease and, based on the results we have presented, the SED would become colder (thanks to the increased dust mass). This effect could introduce a correlation between IR luminosity surface density and effective dust temperature, but it would not indicate that the physical size of the galaxy affects the SED shape (because in the test described above, the half-mass-radius would not change). We defer a detailed exploration of this issue to future work.

\subsection{The Origin of Catastrophic Failures in the SED Prediction}

Figure 7 indicates that most galaxies for which the SED prediction is a catastrophic failure (i.e., $\chi_{r}^{2} \gg 10$ ) have high $L_{\mathrm{AGN}} / L_{\mathrm{IR}}$ and/or $L_{\mathrm{IR}}>10^{12.5} L_{\odot}$. There are a few potential reasons that the SEDs of such sources would prove to be especially difficult to predict. For simulated galaxies in which the AGN contributes significantly to the bolometric luminosity, the AGN can heat host-galaxy dust and cause FIR emission (this will be discussed in detail in C. C. Hayward et al. 2016, in preparation, and E. J. Roebuck et al. 2016, in preparation) Sources in which the AGN dominates the dust heating can differ from star-formation-dominated sources in terms of the SED of the radiation absorbed by the dust. The geometry of such sources may also qualitatively differ: when the AGN dominates, the geometry is more similar to the "shell" geometry of Misselt et al. (2001) than the mixed geometry, whereas the latter should better describe sources in which star formation dominates the dust heating, as argued in the previous subsection. Finally, in the AGN-dominated and most-IRluminous sources, dust self-absorption is likely more significant than in the other sources. Some or all of the above differences between star-formation-powered and AGN-powered IR sources may explain why the SEDs of many of the sources with high $L_{\mathrm{AGN}} / L_{\mathrm{IR}}$ or $/$ and $L_{\mathrm{IR}}>10^{12.5} L_{\odot}$ cannot be predicted well.

\subsection{Implications for IR Counts in Hierarchical Models}

Cosmological galaxy formation models have long struggled to correctly reproduce the observed IR and sub-mm counts without introducing fairly radical assumptions such as an extremely top-heavy stellar initial mass function (Devriendt \& Guiderdoni 2000; Baugh et al. 2005; Davé et al. 2010; Lacey et al. 2010; Niemi et al. 2012; Somerville et al. 2012, but cf. Hayward et al. 2013b; see also the discussion in Casey et al. 2014). Due to the infeasibility of carrying out full 3D radiative transfer calculations on a cosmological hydrodynamic simulation (see 8.6), most such calculations to date have relied 
on SAMs combined with a simplified approach to computing the FIR SEDs. For example, the models of Devriendt \& Guiderdoni (2000) and Somerville et al. (2012) used empirical libraries of dust emission templates parameterized only by $L_{\mathrm{IR}}$. These libraries clearly cannot capture the observed redshift evolution of the relationship between the effective dust temperature and IR luminosity. The SAMs presented by Somerville et al. (2012) and further investigated by Niemi et al. (2012) underpredicted IR number counts at wavelengths $\gtrsim 100 \mu \mathrm{m}$, and the discrepancy was worse with increasing wavelength. Our work here suggests a straightforward and physically motivated way to improve the modeling of dust emission in SAMs by using templates that depend on both $L_{\mathrm{IR}}$ and $M_{\text {dust }}$. Our results are also encouraging for the use of SAMs to model dust emission for large samples of galaxies, as they suggest that the sizes and detailed geometries of galaxies (which are properties that SAMs cannot model accurately) are subdominant compared to the global parameters $L_{\mathrm{IR}}$ and $M_{\text {dust }}$.

If indeed the dust is colder (at fixed $L_{\mathrm{IR}}$ ) in high-redshift galaxies, as indicated by observations, adopting two-parameter templates like the ones we have presented here will help alleviate the tension between the SAM predictions and observations. Accounting for the effects of blending will further reduce the discrepancy (Hayward et al. 2013a, 2013b; Cowley et al. 2015; Muñoz Arancibia et al. 2015). However, it remains to be seen whether SAMs predict a strong enough evolution of $M_{\text {dust }}$ with $L_{\mathrm{IR}}$ and redshift to reproduce the observed IR-sub-mm counts, once observational effects such as blending have been taken into account. We plan to investigate this by incorporating two-parameter dust emission templates in the Somerville et al. (2008) SAM in a future work (M. Safarzadeh et al. 2016, in preparation).

\subsection{Limitations and Future Work}

The detailed simulation methodology that we employed in this work has the advantages of being well studied, and the specific simulations used in this work and similar simulations have previously been demonstrated to reproduce the properties of a wide range of real galaxies, as discussed in Section 1. However, there are some limitations. First, because of the manner in which our sample was constructed, the demographics of the population are by no means representative of those of the real universe. To achieve a cosmologically representative population, it would be necessary to perform $3 \mathrm{D}$ radiative transfer on galaxies selected from a large-volume cosmological simulation. Unfortunately, such simulations typically have spatial resolution $\gtrsim 1 \mathrm{kpc}$ and thus do not resolve galaxy disk scaleheights, much less the internal structure of the ISM. Moreover, state-of-the-art cosmological simulations lack starbursts (i.e., there are significantly fewer outliers above the "star formation main sequence" than observed; Sparre et al. 2015), which are thought to power local ULIRGs and a non-negligible fraction of $z \sim 2-3$ ULIRGs (Hopkins et al. 2010; Hayward et al. 2013a, 2013b; Cowley et al. 2015). Consequently, the utility of such simulations for investigating the FIR SEDs of galaxies remains limited. Cosmological "zoom-in" simulations can be used to achieve orders of magnitude better spatial resolution; thus, radiative transfer can be meaningfully applied to such simulations (e.g., Granato et al. 2015). However, the considerable computational expense of such simulations strongly constrains the subset of the parameter space that can be sampled.

Even for idealized, comparatively simple simulations such as ours, the computational expense required to perform the radiative transfer is significant. Consequently, the parameter space spanned by our simulation suite is not exhaustive, and the sampling of the parameter space is rather coarse. This limitation can be addressed in the future through the use of a (considerably) larger simulation suite or through performing radiative transfer on all resolved galaxies in a large-volume cosmological simulation; however, we again stress that for the latter, the limited spatial resolution will continue to be a significant hurdle for the foreseeable future. We have no reason to expect that our qualitative conclusions would differ if we were to use a larger and/or cosmologically representative simulation suite, especially given the demonstrated agreement between our simulated galaxies' and real galaxies' SEDs. However, it is possible that the details, such as the variation in the SED templates, are sensitive to the specific simulations used.

Another significant limitation is that the hydrodynamical simulations do not resolve the detailed structure of the ISM, both because of the spatial resolution and the ISM model employed. As discussed in Section 2, we assume that the dust is uniformly distributed on sub-resolution scales. Using our current methods, it is possibly to crudely characterize the uncertainty associated with the sub-resolution ISM structure by comparing two extremes: the default model and the one in which the dust in the cold clouds implicit in the Springel \& Hernquist (2003) ISM is completely ignored (i.e., the clumps have a volume filling factor of zero). We performed such a comparison and found that the results do not qualitatively differ. In fact, it is actually easier to predict the SEDs when the latter treatment is used. We speculate that this is because all optical depths are smaller (by construction); consequently, dust self-absorption is less significant.

Moreover, we by no means claim that our treatments of stellar and AGN feedback are state-of-the-art, and various groups are now utilizing more sophisticated and likely more realistic feedback models (e.g., Agertz et al. 2013; Hopkins et al. 2014; Agertz \& Kravtsov 2015). However, to our knowledge, no UV-mm SEDs for such simulations have been presented in the literature; thus, the SEDs used in this work still represent the state-of-the-art for UV-mm SEDs computed by performing dust radiative transfer on hydrodynamical simulations. Computations of UV-mm SEDs of galaxies from the Feedback in Realistic Environments cosmological zoom-in simulations (Hopkins et al. 2014) using Sunrise are underway, but this is a significant undertaking in and of itself. Our method could be applied to these and other SED data sets in the future.

Finally, we demonstrated that PCA is a useful tool for identifying which parameters drive the variation in galaxy SEDs. However, the PCA results cannot be used to predict SEDs of galaxies outside our parameter space because the mean SED and PCs depend on the data set on which the PCA is performed. Moreover, because of the dependence on the mean SED, and the fact that our simulation suite is not cosmologically representative in terms of the galaxy demographics, the PCA results cannot even be used to predict the SEDs for samples in which the parameter space is a subset of that spanned by our simulations, but the distribution within the parameter space differs. Thus, the templates that we provide are 
a better tool for predicting FIR SEDs than are the PCA results. Moreover, other statistical methods, such as neural networks, may also prove to be more useful than PCA for this purpose (e.g., Silva et al. 2012).

\section{CONCLUSIONS}

We performed PCA on a sample of FIR SEDs of simulated galaxies, which we generated by performing dust radiative transfer on hydrodynamical simulations in post-processing. Our goal was to determine what drives the variation in galaxies' FIR SEDs. Our main conclusions are the following.

1. The PCA indicated that only two PCs are sufficient to explain $97 \%$ of the variance in our SED sample. The first component characterizes the peak of the SED, whereas the second characterizes the breadth of the peak.

2. The coefficient of the first PC, $C 1$, is correlated with the IR luminosity, SFR, and AGN luminosity. This result indicates that the SEDs are hotter when the IR luminosity, SFR, or AGN luminosity are greater.

3. Incorporating dust mass increases our ability to predict the value of $C 1$ and thus the FIR SEDs. At fixed IR luminosity, increased dust mass leads to lower $C 1$ values and thus cooler SEDs.

4. The coefficient of the second PC, $C 2$, is weakly anticorrelated with IR luminosity, SFR, AGN luminosity, and dust mass. It can also be predicted using $L_{\mathrm{IR}}$ and $M_{\text {dust }}$, but the dependences on both quantities are weak. Using the second PC improves how well the SEDs can be predicted in some cases, but makes the predictions worse in others.

5. Examination of the catastrophic failures to reconstruct SEDs revealed that the bulk of such SEDs correspond to simulated galaxies with high AGN fractions and/or $L_{\mathrm{IR}}>10^{12.5} L_{\odot}$. For this sample, we were unable to predict the PC coefficients and hence SEDs well.

6. Incorporating galaxy sizes does not improve our ability to predict the SEDs. Previous work (Misselt et al. 2001) has demonstrated that when sources and dust are well mixed, the overall spatial extent of the system does not affect the dust temperature. Because in the simulations (and likely in reality), both star formation and dust density are correlated with gas density, the geometry more resembles such a mixed geometry than a "shell" or "foreground screen" geometry in which the dust surrounds a central source (and for which the size of the system does affect the dust temperature). Thus, our result is consistent with the calculations of Misselt et al. (2001). A review of the literature indicated that the evidence that the correlation between effective dust temperature and IR luminosity surface density is stronger than that between effective dust temperature and IR luminosity is rather weak.

7. These conclusions suggest that the redshift evolution in effective dust temperature (i.e., at fixed $L_{\mathrm{IR}}, z \sim 2-3$ galaxies exhibit lower effective dust temperatures compared with $z \sim 0$ galaxies) is not a consequence of higher-redshift ULIRGs being more extended, as is often suggested. Instead, our work suggests that this difference is driven by $z \sim 2-3$ ULIRGs having higher dust masses at fixed $L_{\mathrm{IR}}$ (because of their higher gas fractions than local galaxies), as suggested by some observations.
8. Because of the importance of dust mass in determining the FIR SED shape, a two-parameter set of IR SED templates that depend on both $L_{\mathrm{IR}}$ and $M_{\text {dust }}$ should be superior to those that depend on $L_{\mathrm{IR}}$ alone. We generated such a set of templates based on our simulated SEDs and made them publicly available. They should be useful for fitting observed galaxy SEDs and predicting galaxy SEDs in unobserved wavelength regimes, and they can be used to predict IR SEDs of galaxies in cosmological simulations and SAMs, as long as the luminosity absorbed by dust and dust mass can be estimated.

We thank Maarten Baes for comments on the manuscript and Brice Menard and Nick Scoville for useful discussions. C.C.H. is grateful to the Gordon and Betty Moore Foundation for financial support. C.C.H. and R.S.S. acknowledge the hospitality of the Aspen Center for Physics, which is supported by the National Science Foundation Grant No. PHY-1066293. R.S.S. is grateful to the Downsbrough family for their support and acknowledges support from the Simons Foundation in the form of a Simons Investigator Award. This work was partially supported by NASA's Astrophysics Data Analysis Program, under grant NNX15AE54G.

\section{REFERENCES}

Agertz, O., \& Kravtsov, A. V. 2015, ApJ, 804, 18

Agertz, O., Kravtsov, A. V., Leitner, S. N., \& Gnedin, N. Y. 2013, ApJ, 770,25

Akaike, H. 1974, ITAC, 19, 716

Armus, L., Mazzarella, J. M., Evans, A. S., et al. 2009, PASP, 121, 559

Baugh, C. M., Lacey, C. G., Frenk, C. S., et al. 2005, MNRAS, 356, 1191

Bernhard, E., Béthermin, M., Sargent, M., et al. 2014, MNRAS, 442, 509

Béthermin, M., Daddi, E., Magdis, G., et al. 2012, ApJL, 757, L23

Béthermin, M., Wang, L., Doré, O., et al. 2013, A\&A, 557, A66

Brassington, N. J., Zezas, A., Ashby, M. L. N., et al. 2015, ApJS, 218, 6

Casey, C. M., Narayanan, D., \& Cooray, A. 2014, PhR, 541, 45

Chakrabarti, S., Cox, T. J., Hernquist, L., et al. 2007, ApJ, 658, 840

Chakrabarti, S., Fenner, Y., Cox, T. J., Hernquist, L., \& Whitney, B. A. 2008 , ApJ, 688, 972

Chakrabarti, S., \& Whitney, B. A. 2009, ApJ, 690, 1432

Chanial, P., Flores, H., Guiderdoni, B., et al. 2007, A\&A, 462, 81

Chapman, S. C., Smail, I., Blain, A. W., \& Ivison, R. J. 2004, ApJ, 614, 671

Chary, R., \& Elbaz, D. 2001, ApJ, 556, 562

Cowley, W. I., Lacey, C. G., Baugh, C. M., \& Cole, S. 2015, MNRAS, 446, 1784

Cox, T. J., Jonsson, P., Somerville, R. S., Primack, J. R., \& Dekel, A. 2008, MNRAS, 384, 386

da Cunha, E., Charlot, S., \& Elbaz, D. 2008, MNRAS, 388, 1595

da Cunha, E., Walter, F., Smail, I. R., et al. 2015, ApJ, 806, 110

Dale, D. A., \& Helou, G. 2002, ApJ, 576, 159

Dale, D. A., Helou, G., Contursi, A., Silbermann, N. A., \& Kolhatkar, S. 2001, ApJ, 549, 215

Davé, R., Finlator, K., Oppenheimer, B. D., et al. 2010, MNRAS, 404, 1355 De Geyter, G., Baes, M., Camps, P., et al. 2014, MNRAS, 441, 869

De Geyter, G., Baes, M., De Looze, I., et al. 2015, MNRAS, 451, 1728

De Looze, I., Baes, M., Fritz, J., \& Verstappen, J. 2012, MNRAS, 419, 895

De Looze, I., Fritz, J., Baes, M., et al. 2014, A\&A, 571, A69

Desert, F.-X., Boulanger, F., \& Puget, J. L. 1990, A\&A, 237, 215

Devriendt, J. E. G., \& Guiderdoni, B. 2000, A\&A, 363, 851

Devriendt, J. E. G., Guiderdoni, B., \& Sadat, R. 1999, A\&A, 350, 381

Domínguez-Tenreiro, R., Obreja, A., Granato, G. L., et al. 2014, MNRAS, 439, 3868

Dopita, M. A., Groves, B. A., Fischera, J., et al. 2005, ApJ, 619, 755

Draine, B. T., Dale, D. A., Bendo, G., et al. 2007, ApJ, 663, 866

Draine, B. T., \& Li, A. 2007, ApJ, 657, 810

Dunne, L., Gomez, H. L., da Cunha, E., et al. 2011, MNRAS, 417, 1510

Dwek, E. 1998, ApJ, 501, 643

Edmunds, M. G., \& Eales, S. A. 1998, MNRAS, 299, L29

Efstathiou, A., Rowan-Robinson, M., \& Siebenmorgen, R. 2000, MNRAS, 313, 734 
Elbaz, D., Dickinson, M., Hwang, H. S., et al. 2011, A\&A, 533, A119

Fritz, J., Franceschini, A., \& Hatziminaoglou, E. 2006, MNRAS, 366, 767

González, J. E., Lacey, C. G., Baugh, C. M., \& Frenk, C. S. 2011, MNRAS, 413,749

Gordon, K. D., Misselt, K. A., Witt, A. N., \& Clayton, G. C. 2001, ApJ, 551,269

Granato, G. L., Lacey, C. G., Silva, L., et al. 2000, ApJ, 542, 710

Granato, G. L., Ragone-Figueroa, C., Domínguez-Tenreiro, R., et al. 2015, MNRAS, 450, 1320

Groves, B., Dopita, M. A., Sutherland, R. S., et al. 2008, ApJS, 176, 438

Hayward, C. C., Behroozi, P. S., Somerville, R. S., et al. 2013a, MNRAS, 434, 2572

Hayward, C. C., Jonsson, P., Kereš, D., et al. 2012, MNRAS, 424, 951

Hayward, C. C., Kereš, D., Jonsson, P., et al. 2011, ApJ, 743, 159

Hayward, C. C., Lanz, L., Ashby, M. L. N., et al. 2014a, MNRAS, 445, 1598

Hayward, C. C., Narayanan, D., Kereš, D., et al. 2013b, MNRAS, 428, 2529

Hayward, C. C., \& Smith, D. J. B. 2015, MNRAS, 446, 1512

Hayward, C. C., Torrey, P., Springel, V., Hernquist, L., \& Vogelsberger, M. 2014b, MNRAS, 442, 1992

Hopkins, P. F., Kereš, D., Oñorbe, J., et al. 2014, MNRAS, 445, 581

Hopkins, P. F., Younger, J. D., Hayward, C. C., Narayanan, D., \& Hernquist, L. 2010, MNRAS, 402, 1693

James, A., Dunne, L., Eales, S., \& Edmunds, M. G. 2002, MNRAS, 335, 753

Jonsson, P. 2006, MNRAS, 372, 2

Jonsson, P., Cox, T. J., Primack, J. R., \& Somerville, R. S. 2006, ApJ, 637, 255

Jonsson, P., Groves, B. A., \& Cox, T. J. 2010, MNRAS, 403, 17

Kelly, B. C., Shetty, R., Stutz, A. M., et al. 2012, ApJ, 752, 55

Kennicutt, R. C., Jr., Armus, L., Bendo, G., et al. 2003, PASP, 115, 928

Kovács, A., Omont, A., Beelen, A., et al. 2010, ApJ, 717, 29

Lacey, C. G., Baugh, C. M., Frenk, C. S., et al. 2010, MNRAS, 405, 2

Lanz, L., Hayward, C. C., Zezas, A., et al. 2014, ApJ, 785, 39

Lanz, L., Zezas, A., Brassington, N., et al. 2013, ApJ, 768, 90

Lee, N., Sanders, D. B., Casey, C. M., et al. 2013, ApJ, 778, 131

Magdis, G. E., Daddi, E., Béthermin, M., et al. 2012, ApJ, 760, 6

Magnelli, B., Lutz, D., Saintonge, A., et al. 2014, A\&A, 561, A86

Magnelli, B., Lutz, D., Santini, P., et al. 2012, A\&A, 539, A155

Martínez-Galarza, J. R., Smith, H. A., Lanz, L., et al. 2014, arXiv:1412.2760

Martini, P., Dicken, D., \& Storchi-Bergmann, T. 2013, ApJ, 766, 121

Michałowski, M. J. 2015, A\&A, 577, A80

Misselt, K. A., Gordon, K. D., Clayton, G. C., \& Wolff, M. J. 2001, ApJ, 551,277
Muñoz Arancibia, A. M., Navarrete, F. P., Padilla, N. D., et al. 2015, MNRAS, 446, 2291

Narayanan, D., Dey, A., Hayward, C. C., et al. 2010a, MNRAS, 407, 1701

Narayanan, D., Hayward, C. C., Cox, T. J., et al. 2010b, MNRAS, 401, 1613

Niemi, S.-M., Somerville, R. S., Ferguson, H. C., et al. 2012, MNRAS, 421, 1539

Pilbratt, G. L., Riedinger, J. R., Passvogel, T., et al. 2010, A\&A, 518, L1

Pope, A., Chary, R.-R., Alexander, D. M., et al. 2008, ApJ, 675, 1171

Popescu, C. C., Tuffs, R. J., Dopita, M. A., et al. 2011, A\&A, 527, A109

Rieke, G. H., Alonso-Herrero, A., Weiner, B. J., et al. 2009, ApJ, 692, 556

Robertson, B., Hernquist, L., Cox, T. J., et al. 2006, ApJ, 641, 90

Rowlands, K., Gomez, H. L., Dunne, L., et al. 2014, MNRAS, 441, 1040

Rujopakarn, W., Rieke, G. H., Eisenstein, D. J., \& Juneau, S. 2011, ApJ, 726, 93

Rujopakarn, W., Rieke, G. H., Weiner, B. J., et al. 2013, ApJ, 767, 73

Scoville, N., Sheth, K., Aussel, H., et al. 2015, arXiv:1505.02159

Siebenmorgen, R., \& Krügel, E. 2007, A\&A, 461, 445

Silva, L., Fontanot, F., \& Granato, G. L. 2012, MNRAS, 423, 746

Silva, L., Granato, G. L., Bressan, A., \& Danese, L. 1998, ApJ, 509, 103

Smith, D. J. B., Hardcastle, M. J., Jarvis, M. J., et al. 2013, MNRAS, 436, 2435

Snyder, G. F., Cox, T. J., Hayward, C. C., Hernquist, L., \& Jonsson, P. 2011, ApJ, 741, 77

Snyder, G. F., Hayward, C. C., Sajina, A., et al. 2013, ApJ, 768, 168

Somerville, R. S., Gilmore, R. C., Primack, J. R., \& Domínguez, A. 2012, MNRAS, 423, 1992

Somerville, R. S., Hopkins, P. F., Cox, T. J., Robertson, B. E., \& Hernquist, L. 2008, MNRAS, 391, 481

Sparre, M., Hartoog, O. E., Krühler, T., et al. 2014, ApJ, 785, 150

Sparre, M., Hayward, C. C., Springel, V., et al. 2015, MNRAS, 447, 3548

Springel, V. 2005, MNRAS, 364, 1105

Springel, V., Di Matteo, T., \& Hernquist, L. 2005, MNRAS, 361, 776

Springel, V., \& Hernquist, L. 2003, MNRAS, 339, 289

Stalevski, M., Fritz, J., Baes, M., Nakos, T., \& Popović, L. Č. 2012, MNRAS, 420, 2756

Symeonidis, M., Vaccari, M., Berta, S., et al. 2013, MNRAS, 431, 2317

Takagi, T., Arimoto, N., \& Hanami, H. 2003, MNRAS, 340, 813

Tateuchi, K., Konishi, M., Motohara, K., et al. 2015, ApJS, 217, 1

Vivian, U, Sanders, D. B., Mazzarella, J. M., et al. 2012, ApJS, 203, 9

Witt, A. N., \& Gordon, K. D. 1996, ApJ, 463, 681

Witt, A. N., \& Gordon, K. D. 2000, ApJ, 528, 799

Wuyts, S., Cox, T. J., Hayward, C. C., et al. 2010, ApJ, 722, 1666

Younger, J. D., Hayward, C. C., Narayanan, D., et al. 2009, MNRAS, 396, L66 